\title{
Real Interference Alignment: Exploiting the Potential of Single Antenna Systems
}

\author{
Abolfazl Seyed Motahari ${ }^{\dagger}$, Shahab Oveis-Gharan ${ }^{\dagger}$, \\ Mohammad-Ali Maddah-Ali ${ }^{\dagger \dagger}$, and Amir Keyvan Khandani ${ }^{\dagger}$ \\ $\dagger$ Department of Electrical and Computer Engineering, University of Waterloo \\ Waterloo, ON, Canada N2L3G1 \\ \{abolfazl,shahab,khandani\}@ cst.uwaterloo.ca \\ ${ }^{\dagger \dagger}$ Department of Electrical Engineering and Computer Sciences, University of California-Berkeley \\ Berkeley, CA, USA \\ maddah-a@eecs.berkeley.edu
}

\begin{abstract}
In this paper, the available spatial Degrees-OfFreedoms (DOF) in single antenna systems is exploited. A new coding scheme is proposed in which several data streams having fractional multiplexing gains are sent by transmitters and interfering streams are aligned at receivers. Viewed as a field over rational numbers, a received signal has infinite fractional DOFs, allowing simultaneous interference alignment of any finite number of signals at any finite number of receivers. The coding scheme is backed up by a recent result in the field of Diophantine approximation, which states that the convergence part of the Khintchine-Groshev theorem holds for points on nondegenerate manifolds. The proposed coding scheme is proved to be optimal for three communication channels, namely the Gaussian Interference Channel (GIC), the uplink channel in cellular systems, and the $X$ channel. It is proved that the total DOF of the $K$-user GIC is $\frac{K}{2}$ almost surely, i.e. each user enjoys half of its maximum DOF. Having $K$ cells and $M$ users within each cell in a cellular system, the total DOF of the uplink channel is proved to be $\frac{K M}{M+1}$. Finally, the total DOF of the $X$ channel with $K$ transmitters and $M$ receivers is shown to be $\frac{K M}{K+M-1}$.
\end{abstract}

Index Terms-Interference channels, interference alignment, number theory, Diophantine approximation.

\section{INTRODUCTION}

$\mathbf{T}$ IME, frequency, and space are natural resources in wireless systems. While time and frequency are two global resources independent of systems' topologies, space is a local resource related to the number of antennas incorporated in transceivers. Spectrum sharing is known as a key solution to time/frequency allocation among several users. To avoid interference in the system, orthogonal schemes do not allow different transmissions overlap in time or frequency. Orthogonal schemes fall short of achieving high throughput in dense networks because allowing for multi-user interference is proved to be optimal in such networks.

Achieving the optimum throughput of a system requires efficient interference management. Interference alignment is a type of interference management that exploits spatial DegreesOf-Freedoms (DOF) available at transmitters and receivers.

\footnotetext{
${ }^{0}$ Financial support provided by Nortel and the corresponding matching funds by the Natural Sciences and Engineering Research Council of Canada (NSERC), and Ontario Ministry of Research \& Innovation (ORF-RE) are gratefully acknowledged.
}

Interference alignment makes the interference less damaging by merging the communication dimensions occupied by interfering signals. In [2], Maddah-Ali, Motahari, and Khandani introduced the concept of interference alignment and showed its capability in achieving the full Degrees-Of-Freedom (DOF) for certain classes of two-user $X$ channels. Being simple and at the same time powerful, interference alignment provided the spur for further research. Besides lowering the harmful effect of the interference, interference alignment can be applied to provide security in networks, c.f. [8].

The study of interaction between two users sharing the same channel goes back to Shannon's work on the two-way channel in [1]. His work was followed by several researchers and the two-user interference channel emerged as the fundamental building block in dealing with interference in networks.

Although partial capacity results on the interference channel are recently derived, c.f. [5]-[7], the problem of characterizing the capacity region of the Gaussian Interference Channel (GIC) is still open. In [4], it is shown that in the two-user GIC, the Han-Kobayashi (HK) scheme [3] achieves within one bit of the capacity region, as long as the interference from the private message in the $\mathrm{HK}$ scheme is designed to be below the noise level.

It turns out that moving from the two-user scenario to a larger number of users is a challenging task. Indeed, for $K$ user GIC $(K>2)$, the Han-Kobayashi approach of interference management is not enough and we need to incorporate the interference alignment in the signaling.

Interference alignment in $n$-dimensional Euclidean spaces for $n \geq 2$ is studied by several researchers, c.f. [2], [9]-[11]. In this method, at each receiver a subspace is dedicated to interference, then the signaling is designed such that all the interfering signals are squeezed in the interference sub-space. Such an approach saves some dimensions for communicating desired signal, while keeping it completely free from the interference. Using this method, Cadambe and Jafar showed that, contrary to the popular belief, a $K$-user Gaussian interference channel with varying channel gains can achieve its total DOF, which is $\frac{K}{2}$. Later, in [12], it is shown that the same result can be achieved using a simple approach based on a particular pairing of the channel matrices. The assumption of varying 
channel gains, particularly noting that all the gains should be known at the transmitters, is unrealistic, which limits the application of these important theoretical results in practice. This paper aims to remove this shortcoming.

In [13], followed up by [14], [15], interference alignment is applied in single antenna systems. In [13], it is shown that lattice codes, rather than random Gaussian codes, are essential parts of signaling for three-user time-invariant GICs. In [14], after aligning interference using lattice codes, the aggregated signal is decoded and its effect is subtracted from the received signal. In fact, [14] shows that the very strong interference region of the $K$-user GIC is strictly larger than the corresponding region when alignment is not applied. In their scheme, to make the interference less severe, transmitters use lattice codes to reduce the code-rate of the interference, which guarantees decodability of the interference at the receiver. In [15], Sridharan et al. showed that the DOF of a class of 3-user GICs with fixed channel gains can be greater than 1 . This result was obtained using layered lattice codes along with successive decoding at the receiver.

In [16] and [17], the results from the field of Diophantine approximation in Number Theory are used to show that interference can be aligned using properties of rational and irrational numbers and their relations. They showed that the total DOF of some classes of time-invariant single antenna interference channels can be achieved. In particular, Etkin and Ordentlich in [16] proposed an upper bound on the total DOF, which accounts for the properties of channel gains with respect to being rational or irrational. Using this upper bound, surprisingly, they proved that the DOF is everywhere discontinuous for the class of channels under investigation.

The channels considered in [16] and [17] are special in the sense that signals not intended for a given receiver are aligned by the channel. Therefore, signaling design is not required due to the nature of the channel. The first example of interference alignment in one-dimensional spaces, which requires signaling design, is presented in [18]. Using irrational numbers as transmit directions and applying Khintchine-Groshev theorem, [18] shows the two-user $X$ channel achieves its total DOF. This is the first channel in which no variations in coefficients over time or frequency and no multiple antennas are required to achieve the total DOF. This is because rational dimensions in one-dimensional spaces can play the role of real dimensions in more-than-two dimensional spaces. In this paper, we take one step forward and prove that the total DOF of the $K$-user GIC, the uplink channel in cellular systems, and the $X$ channel can be achieved without the need for channel variation over time/frequency/space.

This paper is organized as follows: in Section III the main theorem of this paper is stated and some discussions follow. In Section III] the main ideas incorporated in the proposed coding scheme are presented. Moreover, several examples are provided to shed light on the ideas. In Section IV, some background on the field of Diophantine approximation and, in particular, Khintchine-Groshev type theorems are presented. Section $\nabla$ describes the coding scheme used to prove the main theorem. Moreover, the performance analysis, based on recent results in the field of Diophantine approximation, is presented.
In Section VI-C, the total DOF of the $K$-user GIC is derived. In Section VII, it is proved that the uplink channel in cellular systems has $\frac{K M}{M+1}$ DOF, where $K$ is the number of cells and $M$ is the number of users within each cell. In Section VIII, the total DOF of the $K \times M X$ channel is derived. Finally, Section $\amalg X$ concludes the paper.

Notation: $\mathbb{R}, \mathbb{Q}, \mathbb{N}$ represent the set of real, rational, and nonnegative integers, respectively. For a random variable $X$, $E[X]$ denotes the expectation value. $(a, b)_{\mathbb{Z}}$ denotes the set of integers between $a$ and $b$.

\section{Main Contributions And Discussions}

\section{A. Main Results}

In this paper, the total DOFs of three channels, namely the $K$-user GIC, the uplink channel in cellular systems, and the $K \times M X$ channel, are characterized using a new coding scheme.

Theorem 1: The total DOF of the $K$-user GIC with real and time invariant channel coefficients is $\frac{K}{2}$ for almost all channel realizations.

Theorem 2: The total DOF of a cellular system consisting of $K$ cells and $M$ users within each cell is $\frac{K M}{M+1}$ for almost all channel realizations.

Theorem 3: The total DOF of the $K \times M X$ channel with real and time invariant channel coefficients is $\frac{K M}{K+M-1}$ for almost all channel realizations.

\section{B. Real Interference Alignment}

The available DOF of the systems having multiple-antenna, time-varying, and/or frequency-selective channels can be efficiently exploited by choosing appropriate signaling directions to maximize the channel gains and avoiding or aligning interference. We refer to the alignment scheme incorporating directional signaling as vector alignment. In contrary, it was commonly believed that time-invariant frequency-flat singleantenna channels are restrictive in the sense that they prevent us to incorporate vector alignment. Here, we develop a machinery that transforms the single-antenna systems into pseudo multiple-antenna systems with infinite-many pseudo antennas. Indeed the number of available dimensions in the resultant pseudo multiple-antenna systems is, roughly speaking, as many as rationally-independent irrational numbers. We see that the pseudo multiple-antenna channels mimics the behavior of real multi-dimensional systems (in time/frequency/space) and, for example, allows us to simultaneously align interference at all receivers of static single-antenna channels. We refer to the alignment scheme applicable in single antenna systems as real alignment.

\section{Almost All vs All Cases}

In the statement of the theorem, it is emphasized that the total DOFs of the $K$-user GIC, the uplink channel in a cellular system, and the $X$ channel are achievable for almost all channel realizations. It means the collection of all possible channel realizations in which the total DOF may not be achieved has measure zero. In other words, if all channel 
gains are drawn independently from a random distribution then almost surely the channel has the desired properties required for achieving the total DOF.

In the case of the $K$-user GIC if all channel gains are rational, then the total DOF is strictly less than $\frac{k}{2}$. This is due to the recent upper bound on the total DOF obtained by Etkin and Ordentlich in [16]. This result, together with Theorem 1 implies that the total DOF of the channel is everywhere discontinuous with respect to channel coefficients. This is due to the fact that for any set of channel gains one can find a set of rational numbers arbitrarily close to it. This behavior is unique to this channel (or related networks with single antennas). In fact, almost all of the total DOFs obtained for Multiple Input Multiple Output (MIMO) systems are discontinuous at a point or on a set of measure zero. However, none of them are everywhere discontinuous.

Other than rational channel gains, infinitely many channel realizations are not covered by the theorems. However, it cannot be concluded that for these realizations the total DOFs are not achievable. In fact, it is proved that there are some cases where the total DOFs can be achieved and those cases are out of the scope of the theorems, c.f., [16]-[18]. As an example, the total DOF of the $K$-user GIC can be achieved by using a single layer constellation at transmitters in the special case where all cross gains are rational numbers and all direct gains are algebraic irrationals (this is the case for almost all irrationals) [16]. This is due to the fact that cross gains lie on a single rational dimension and therefore, the effect of the interference caused by several transmitters behaves as that of interference caused by a single transmitter. Using a single data stream, one can deduce that the multiplexing gain of $\frac{1}{2}$ is achievable for each user.

\section{Time Varying versus Time-Invariant Channels}

Cadambe and Jafar in their papers [10] and [11] proved that the total DOFs of the time-varying $K$-user GIC and $X$ channel can be achieved. They showed that the variation of the channel in time, if it is fast enough to be assumed independent, provides enough freedom to align the interference. However, such an assumption about the variation of wireless channels is not practically realistic. Moreover, it imposes an inadmissible delay on the system, noting that wireless channels are changing slowly.

Here, we propose a signaling scheme that achieves the total DOFs in almost all realizations of the channel without imposing any delay to the system or requiring channel variation. Indeed, the channel can be static over time and still it is possible to achieve the total DOFs of the channels.

\section{E. MIMO and Complex Coefficients Cases}

Let us consider the $K$-user MIMO GIC where each node in the network is equipped with $M$ antennas. The upper bound on the total DOF states that at most $\frac{M K}{2}$ is achievable for this channel. Except for the three-user case where Cadambe and Jafar in [10], through explicit interference alignment, showed that $\frac{3 M}{2}$ is achievable, the total DOF of $K$-user MIMO GIC with static channel states is not considered in the literature.
Again, if we assume time-variant channels, however, this upper bound can be achieved, see [10].

The applicability of Theorem 1 is not restricted to the single antenna case. In fact, we can also show that for the $K$-user MIMO GIC the total DOF of the channel can be achieved for almost all cases. This can be proved by simply viewing a single user as $M$ virtual users in which a transmit antenna is paired with a receive antenna. Using separate encoding (resp. decoding) at all transmit (resp. receive) antennas, the channel becomes a $M K$-user single antenna GIC. Applying the theorem to this channel, we conclude that the total of $\frac{M K}{2}$ is achievable and this meets the upper bound. In [21]), the total DOF of the $K$-user IC is obtained for the case where the numbers of transmit and receive antennas are different.

Needless to say, Theorem 1 is also applicable to channels (either single or multiple antennas) with complex coefficients. In fact, the real and imaginary parts of the input and the output can be paired. This converts the channel to $2 K$ virtual users. Therefore, the total DOF of the channel can be achieved by a simple application of the theorem. It is worth noting that joint processing between all antennas and/or real-imaginary parts at a transmitter increases the achievable sum rate of the channel. However, at high SNR regimes this increase vanishes and the total DOF of the channel can be achieved by separate coding over all available dimensions.

The total DOF of the $X$ channel with complex coefficients follows similar behavior, but it can not be derived by pairing. In fact, a simple extension of the coding proposed in this paper results in the total DOF of this channel [19].

\section{MAIN IDEAS AND BASIC EXAMPLES}

In this section, we review some important features of the real interference alignment introduced in [18] and extend its application to more general cases. To clarify basic ideas, we rely on some simple examples and provide only rough reasoning for rationality of the schemes. Unless otherwise stated, the following assumptions are in place throughout this section.

\section{Generic Assumptions}

1) All channels are additive.

2) The received signals are corrupted by unit variance additive Gaussian noise.

3) All transmitters are subject to power constraint $P$.

In [18], constellation points carved from integers are used to construct a code for transmission of a given data stream. Carrying multiple data streams, a transmitter designs its transmit constellation based on a linear combination of constellations designed for individual data streams. Since all transmitters use a set of finite points as the input symbols, the received symbol before corruption by additive noise is also a finite set, which is called the received constellation.

It will be shown that the performance of the system is highly related to the design of transmit constellations. In order to focus on the important aspects of the optimum constellation 


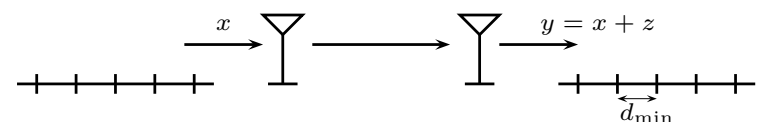

Fig. 1. A point-to-point communication system. The receive constellation is the same as the transmit constellation.

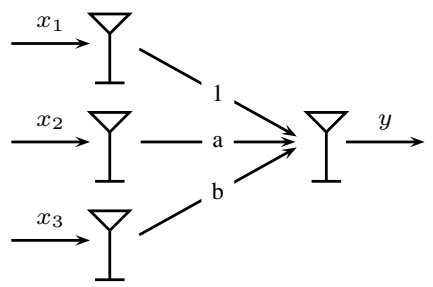

Fig. 2. A multiple access channel.

design, we bypass the effect of error correction codes and assume that receivers can remove the additive noise under the following condition:

\section{Noise Removal}

$A$ receiver can completely remove the noise if the minimum distance of the received constellation points is greater than $\sqrt{N}$, where $N$ is the noise variance.

The preceding assumption is by no means correct. However, it provides accurate estimates of the total DOFs of the systems under investigation. In the following sections, we will explain that if the minimum distance of the received constellation is of order of $\sqrt{N} P^{\epsilon}$ for any $\epsilon>0$, then a code with rate arbitrary close to the size of the transmit constellation exists such that the noise can be completely removed from the received signal. To see the power of the above assumption, we look at the following examples.

Example 1 (Point-to-point communication): A single user channel is shown in Figure 1 Given an integer $Q$, the transmit constellation $\mathcal{U}=(-Q, Q)_{\mathbb{Z}}=\{-Q,-Q+1, \ldots, Q-1, Q\}$ is used for transmission of a single data stream. Since it is assumed that the additive noise has unit variance and the minimum distance in the received constellation, which is the same as the transmit constellation, is also one, the noise can be removed from the received signal. Therefore, $R \approx \log (2 Q-1)$ is achievable for the channel. On the other hand, the input power is less than $Q^{2}$. Hence, $P=Q^{2}$. The multiplexing gain associated with the data stream can be computed as

$$
r=\lim _{P \rightarrow \infty} \frac{R}{0.5 \log P}=1 \text {. }
$$

Example 2 (Multiple Access Channel): A multiple access channel with three users is shown in Figure 2 The channel can be modeled as

$$
y=x_{1}+a x_{2}+b x_{2}+z .
$$

It is assumed that $a$ and $b$ are two real numbers. Moreover, let us assume that all three users communicate with the receiver using a single data stream. The data streams are modulated by the constellation $\mathcal{U}=A(-Q, Q)_{\mathbb{Z}}$ where $A$ is a factor controlling the minimum distance of the received constellation.
The received constellation consists of points representable by $A\left(u_{1}+a u_{2}+b u_{3}\right)$ where $u_{i}$ s are integer. Let us choose two distinct points $v_{1}=A\left(u_{1}+a u_{2}+b u_{3}\right)$ and $v_{2}=A\left(u_{1}^{\prime}+\right.$ $\left.a u_{2}^{\prime}+b u_{3}^{\prime}\right)$ in the received constellation. The distance between these two points is $d=A \mid\left(u_{1}-u_{1}^{\prime}\right)+a\left(u_{2}-u_{2}^{\prime}\right)+b\left(u_{3}-\right.$ $\left.u_{3}^{\prime}\right) \mid$. Khintchine-Groshev theorem (see Section IV] Theorem 4) provides us a lower bound on any linear combination of integers. Using the theorem, one can obtain $d_{\text {min }} \approx \frac{A}{Q^{2}}$ where $d_{\text {min }}$ is the minimum distance in the received constellation. To be able to remove the noise, $d_{\min }=1$. Hence, $A \approx Q^{2}$. In a noise-free environment, the receiver can decode the three messages if there is a one-to-one map from the received signal to the transmit constellation. Mathematically, one can satisfy this condition by enforcing the following:

\section{Separability Condition}

The receiver is able to decode the three messages if $a$ and $b$ are rationally independent. In other words, $p_{1}+a p_{2}+b p_{3}=0$ has no non-trivial solution in integers $p_{1}, p_{2}$, and $p_{3}$.

Having the above condition, the receiver can decode all three messages. To calculate User $i$ 's rate $R_{i}=\log (2 Q-1)$ in terms of $P$, we need to find a relation between $Q$ and $P$. Due to the power constraints, we have $P=A^{2} Q^{2}$. We showed that $A \approx Q^{2}$. Therefore, $P \approx Q^{6}$. Hence, we have

$$
r_{i}=\lim _{P \rightarrow \infty} \frac{R_{i}}{0.5 \log P}=\frac{1}{3} .
$$

Two facts are hindered in the preceding example. First, Khintchine-Groshev theorem is not valid for all possible values of $a$ and $b$. In fact, there are infinitely many cases that are not addressed in the theorem (see Figure 3). However, the theorem asserts that the measure of these points is zero. In other words, for any smooth probability distribution on the pair of $(a, b)$, the probability that the theorem holds is one. Second, the separability condition does not hold in general. In fact, this condition holds again with measure one. Hence, we can conclude:

\section{Achievablity for Almost All Cases}

The proofs presented in this paper are based
on the separability condition and Khintchine-
Groshev type theorems. Therefore, all results are
valid for almost all channel realizations.

As mentioned in the previous example, the pair $(a, b)$ can possibly take all vectors in $\mathbb{R}^{2}$. Let us assume that $a$ and $b$ have a relation. For instance, $b$ is a function of $a$, say $b=a^{2}$. In this case, the pair $(a, b)$ lies on a one dimensional manifold in $\mathbb{R}^{2}$, see Figure 3 Since the manifold itself has measure zero, Khintchine-Groshev theorem can not be applied directly. For such cases, however, there is an extension to KhintchineGroshev theorem (see Section IV. Theorem 5) which states that the same lower bound on the minimum distance can be applied when coefficients lie on a non-degenerate manifold and, in fact, the measure of points not satisfying the theorem is zero. 


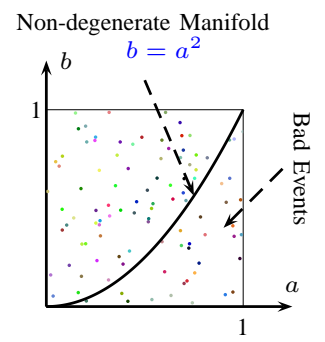

Fig. 3. There are infinitely many points on the $a-b$ plane with measure zero that are not addressed by the Khintchine-Groshev theorem (these are called bad events). The curve $b=a^{2}$ is a non-degenerate manifold and by the extension of Khintchine-Groshev theorem, the measure of bad events is zero on the curve.

Non-degenerate Manifolds [25]

Let $U \subset \mathbb{R}^{d}$ be an open set. The function $f$ :

$U \rightarrow \mathbb{R}^{n}$ is l-non-degenerate at $x_{0} \in U$ if

1) $f$ is l times continuously differentiable on some sufficiently small ball centered at $x_{0}$.

2) Partial derivatives of $f$ at $x_{0}$ of orders up to l span $\mathbb{R}^{n}$.

The function $f$ is non-degenerate at $x_{0}$ if it is $l$-non-degenerate at $x_{0}$ for some $l \in N$. We say that $f$ is non-degenerate if it is non-degenerate almost everywhere on $U$.

The preceding example can be extended to the multi-user multiple access channel. The following statement presents the result:

\section{Achievable DOF in a Multiple Access Channel}

In a multiple access channel with $K$ users, each user enjoys $\frac{1}{K}$ of the total DOF provided that the channel coefficients are independent over rational numbers and lie on a non-degenerate manifold in $\mathbb{R}^{K}$.

Remark 1: Due to the general statement of the achievablity for almost all cases, the preceding statement about the total DOF of the $K$-user multiple access channel holds for almost all channels. However, the capacity region of the $K$-user multiple access channel is completely characterized and it can be shown that under all circumstances each user can enjoy $\frac{1}{K}$ of the total DOF. We will show that the above coding scheme is capable of achieving the total DOF of channels with interference.

In the following example, we will look at the two-user $X$ channel that was originally introduced in [2]. This example adds two important features to the signaling. First, multiple data streams are transmitted from each transmitter. Second, interference alignment is required to achieve the total DOF of the channel.

Example 3 (Two-user X Channel [18]): In the two-user $X$ channel, each transmitter has independent messages to both receivers, see Figure 4 Hence, each transmitter has two data streams and they need to be transmitted such that they can be separated in their corresponding receivers. In [18], the

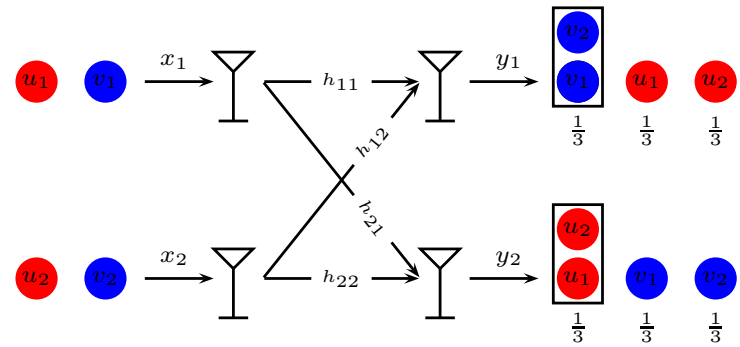

Fig. 4. The two-user $X$ channel. Data streams intended for the first receiver, $u_{1}$ and $u_{2}$, are aligned at the second receiver occupying one third of the received dimension. Similarly, data streams intended for the second receiver, $v_{1}$ and $v_{2}$, are aligned at the first receiver occupying one third of the received dimension.

following signaling is proposed for the channel:

$$
\begin{aligned}
& x_{1}=h_{22} u_{1}+h_{12} v_{1}, \\
& x_{2}=h_{21} u_{2}+h_{11} v_{2},
\end{aligned}
$$

where $u_{1}, u_{2}$ and $v_{1}, v_{2}$ are data streams intended for the first and second receivers, respectively. All data streams are transmitted using the constellation $\mathcal{U}=A(-Q, Q)_{\mathbb{Z}}$, where $Q$ is an integer and $A$ is the factor controlling the minimum distance of the received constellation.

The direction used to transmit data streams are chosen based on the channel coefficients. As we will explain, this choice allows us to align unwanted signals at receivers. In general, we can state the following:

\section{Transmit Directions Matching the Channel}

To transmit multiple data streams from a transmitter, channel coefficients are used as generators for the directions.

Using the above signaling, the received signal can be written as:

$$
\begin{aligned}
& y_{1}=\left(h_{11} h_{22}\right) u_{1}+\left(h_{21} h_{12}\right) u_{2}+\left(h_{11} h_{12}\right)\left(v_{1}+v_{2}\right)+z_{1}, \\
& y_{2}=\left(h_{21} h_{12}\right) v_{1}+\left(h_{11} h_{22}\right) v_{2}+\left(h_{21} h_{22}\right)\left(u_{1}+u_{2}\right)+z_{2} .
\end{aligned}
$$

The received signals are linear combinations of three terms in which two of them are the intended data streams and one is the sum of interfering signals, see Figure 4, Let us focus on the first receiver. $y_{1}$ resembles the received signal of a multiple access channel with three users. However, there is an important difference between them. In the two-user $X$ channel the term corresponding to the interfering signals, i.e. $u_{3}=v_{1}+v_{2}$, is a sum of two data streams. However, we claim that this difference does not change considerably the minimum distance of the received constellation, i.e. $d_{\min }$. Recall that KhintchineGroshev theorem is used to bound $d_{\min }$. The bound is a function of the maximum value that the integers can take. The maximum value of $u_{3}$ is $2 A Q$, which is different from a single data stream by a factor of two. Since this change only affects the constant term of Khintchine-Groshev theorem, we have $d_{\min } \approx \frac{A}{Q^{2}}$ and the receiver can decode all data streams if each of them have a multiplexing gain of $\frac{1}{3}$. Therefore, the multiplexing gain of $\frac{4}{3}$ is achievable in total, which meets the upper bound. 
Interference Alignment

Two data streams are aligned at a receiver if

they arrive at the receiver with the same received

direction (coefficient).

It is interesting to see what Khintchine-Groshev theorem offers when $v_{1}$ and $v_{2}$ receive in different directions. In this case, the received constellation consists of points representable by a linear combination of four integers. Therefore, KhintchineGroshev theorem gives us $d_{\min } \approx \frac{A}{Q^{3}}$. Hence, each data stream can carry information with a multiplexing gain of $\frac{1}{4}$, and in total, the DOF of 1 is achievable. This means interference alignment reduces the power of $Q$ in the expression of $d_{\min }$, which in turn allows achieving higher DOFs.

The signaling proposed for the two-user $X$ channel can be interpreted as follows. The received signal at each receiver is a real number, which is a one-dimensional component. One can embed three rational dimensions, each of which has dimension $\frac{1}{3}$ in this one dimensional space, see Figure 4 One of these dimensions is associated with interference and the other two with intended signals. Therefore, $\frac{4}{3}$ out of two dimensions available at both receivers are used for data, which in turn gives us the total DOF of the channel. In general, we can state:

\section{Rational Dimension Occupation}

If a receiver observes $K$ data stream in $K$ different directions, then each data stream occupies $\frac{1}{K}$ of the receiver's dimension. Moreover, if multiple data streams are aligned at a receiver then the dimension that they occupy is the same as that of a single data streams.

As above example reveals, available dimensions at all receivers, like time and frequency, are natural resources in wireless systems. Interference alignment at all receivers is a way of exploiting the full potential of these resources by reducing the unused dimensions at all receivers. In the two user $X$ channel, we have observed that interfering signals from two different sources can be easily aligned at a single receiver. Moreover, two interfering streams are received with the same direction occupying only $\frac{1}{3}$ of the available dimensions of the receivers. This is in fact the best efficiency that one can hope for in reducing the number of waste dimensions. This idea inspires us to define the alignment efficiency as follows.

\section{Alignment Efficiency}

Let us consider that all transmitters transmit the same number of data streams, say $L_{t}$, using $L_{t}$ different directions. Moreover, the maximum dimension occupied by interference at all receivers is caused by $L_{r}$ received directions. The alignment efficiency $\eta$ is defined as the ratio of $L_{t}$ and $L_{r}$ as

$$
\eta=\frac{L_{t}}{L_{r}}
$$

Alignment is called perfect if $\eta=1$.
In the two user $X$ channel, we were able to perform perfect alignment in the system. As the following example shows, however, this is not the case in general.

Example 4 (Alignment at two receivers): Let us consider a communication scenario in which three transmitters try to align their signals at two different receivers. The channel is depicted in Figure 5 In order to shed light on the alignment part of the signaling, the intended receivers are removed from the picture.

Alignment can be done at the first receiver by sending a single data stream with direction 1 from each of the transmitters; whereas alignment at the second receiver requires $b c$, $a c$, and $a b$ as chosen transmit directions for first, second, and third transmitters, respectively. In general, it is not possible to simultaneously align three single data streams at two different receivers. Therefore perfect alignment is not feasible by transmitting single data streams from each transmitter.

The solution to this problem is partial alignment, which is first introduced in [10]. In this technique, instead of sending just one data stream, several data streams are transmitted from each transmitter. The idea is to choose the transmit directions based on channel coefficients in such a way that the number of received directions is minimum. For sake of simplicity, we choose the same directions at all transmitters. Let $\mathcal{T}$ denote the set of transmit directions. A direction $T \in \mathcal{T}$ is chosen as a transmit direction if it can be represented as

$$
T=a^{s_{1}} b^{s_{2}} c^{s_{3}},
$$

where $0 \leq s_{i} \leq n-1$ for all $i \in\{1,2,3\}$. In this way, the total number of transmit directions is $L_{1}=n^{3}$.

\section{Generating Transmit Directions}

Let $G=\left\{g_{1}, g_{2}, \ldots, g_{m}\right\}$ denote a finite set of real numbers. The set of transmit directions $\mathcal{G}$ generated by $G$ is the collection of all real numbers representable by

$$
g_{1}^{s_{1}} g_{2}^{s_{2}} \cdots g_{m}^{s_{m}},
$$

where $s_{i} \in \mathbb{N}$ for all $i \in\{1,2, \ldots, m\}$. $\mathcal{G}$ is closed under multiplication.

To compute the efficiency of the alignment, one needs to find the set of received directions in the first and second receivers, which are denoted by $\mathcal{T}_{1}$ and $\mathcal{T}_{2}$, respectively. Since all transmit directions arrive at the first receiver intact, $\mathcal{T}_{1}=\mathcal{T}$.

To compute the set of received directions at the second receiver, we look at the received directions due to the first, second, and third transmitters separately. Since all of them are multiplied by $a$, the received directions due to the first transmitter are of the form $a^{s_{l}+1} b^{s_{2}} c^{s_{3}}$, where $0 \leq s_{i} \leq n-1$ for all $i \in\{1,2,3\}$. Similarly, $a^{s_{l}} b^{s_{2}+1} c^{s_{3}}$ and $a^{s_{l}} b^{s_{2}} c^{s_{3}+1}$ are the types of received directions due to the second and third transmitter, respectively. Taking the union of all these directions, one can compute $\mathcal{T}_{2}$. However, we can easily see that the set of directions formed by $a^{s_{l}} b^{s_{2}} c^{s_{3}}$, where $0 \leq$ $s_{i} \leq n$ for all $i \in\{1,2,3\}$ includes $\mathcal{T}_{2}$ and can be used as an upper bound on the number of received directions. This 


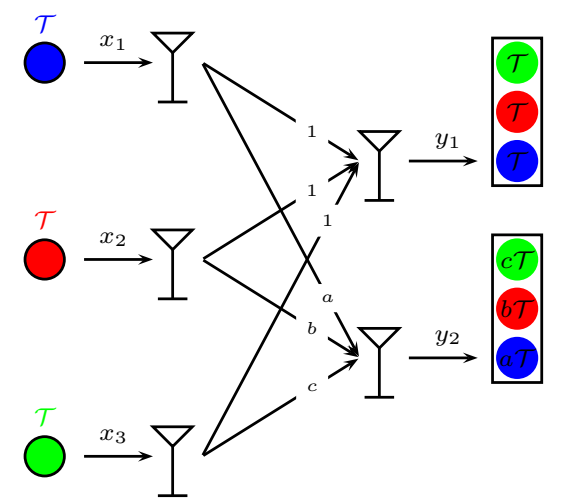

Fig. 5. Three transmitters wish to align their signals at two receivers. Each circle on the transmitters' sides represents a set of data streams transmitted in the directions $\mathcal{T}$. Each circle on the receivers' sides represent the received directions due to different transmitters. The received directions can be aligned with efficiency arbitrary close to one.

set has $(n+1)^{3}$, which is an upper bound for $L_{2}$. Hence, we conclude that

$$
\eta=\frac{L_{1}}{L_{2}}>\left(\frac{n}{n+1}\right)^{3} .
$$

Since $n$ is an arbitrary integer, any alignment efficiency close to 1 is possible. Hence, the partial alignment approaches the perfect alignment.

For the multiple transmitter and receiver, the above approach can be easily extended. In fact, it can be shown that the perfect alignment is possible for any finite number of transmitters and receivers.

\section{Simultaneous Interference Alignment}

Partial interference alignment of any finite number of signals is possible at any finite number of receivers. Moreover, by increasing the number of transmit directions, one can achieve any alignment efficiency close to one.

The above example shows that a set of data streams with the appropriate directions in any system can play the role of a single data stream in the two-user $X$ channel, where perfect interference alignment was possible. In addition to perfect alignment, which is desired in any system, receivers are required to decode their own messages from the received signals. However, the receiver can decode its own messages if the intersection of the set of received directions due to interference and message is null.

In the last example, we will combine all ideas presented in this section to obtain the total DOF of the $3 \times 3 X$ channel.

Example $5(3 \times 3 X$ channel $)$ : In this example, we consider the $3 \times 3 X$ channel shown in Figure 6 In this channel, each transmitter has independent messages for all three receivers. Let $m_{j i}$ denote the message transmitted by the $i$ th transmitter and intended for the $j$ th receiver, where $i, j \in\{1,2,3\}$. In addition, let $x_{i j}$ denote the signal carrying the message $m_{j i}$.

The transmitters send their messages using the following signaling:

$$
\begin{aligned}
& x_{1}=h_{11} x_{11}+h_{21} x_{21}+h_{31} x_{31}, \\
& x_{2}=h_{12} x_{12}+h_{22} x_{22}+h_{32} x_{32}, \\
& x_{3}=h_{13} x_{13}+h_{23} x_{23}+h_{33} x_{33} .
\end{aligned}
$$

The messages intended for the first receiver are transmitted by $x_{11}, x_{12}$, and $x_{13}$ (red circles in Figure 6). In the previous example, we have shown that the signals carrying these data can be efficiently aligned at the second and third receivers using transmit directions $\mathcal{G}_{1}$ generated by

$$
G_{1}=\left\{h_{11} h_{21}, h_{11} h_{31}, h_{12} h_{22}, h_{12} h_{32}, h_{13} h_{23}, h_{13} h_{33}\right\} .
$$

To see this, let us consider the signal $x_{11}$. This signal arrives at the second and third receivers multiplied by $h_{11} h_{21}$ and $h_{11} h_{31}$, respectively. In other words, $h_{11} h_{21}$ and $h_{11} h_{31}$ are equivalent channel gains between $x_{11}$ and the second and third receivers. Therefore, these factors need to be incorporated in the selection of transmit directions to have efficient alignment at both receivers. A similar argument can be applied for $x_{12}$ and $x_{13}$. In a similar fashion, one can obtain the sets $\mathcal{G}_{2}$ and $\mathcal{G}_{3}$ used for sending messages to the second and third receivers by using generators

$$
G_{2}=\left\{h_{21} h_{11}, h_{21} h_{31}, h_{22} h_{12}, h_{22} h_{32}, h_{23} h_{13}, h_{23} h_{33}\right\}
$$

and

$$
G_{3}=\left\{h_{31} h_{11}, h_{31} h_{21}, h_{32} h_{12}, h_{32} h_{22}, h_{33} h_{13}, h_{33} h_{23}\right\},
$$

respectively.

The previous example ensures us that the preceding signaling is efficient regarding interference alignment. However, we need to guarantee that the messages can be decoded at the intended receivers. To this end, we look at the received directions at the first receiver. $h_{11}^{2} \mathcal{G}_{1}, h_{12}^{2} \mathcal{G}_{1}$, and $h_{13}^{2} \mathcal{G}_{1}$ are received directions due to the intended messages. Clearly, they are all different and therefore can be separated based on the separability condition. Moreover, it can be shown that the set of intended directions has no intersection with the set of interfering directions represented by $\mathcal{G}_{2} \cup \mathcal{G}_{3}$ (recall that $\mathcal{G}_{2}$ and $\mathcal{G}_{3}$ are closed under multiplication). Dividing the dimension of the first receiver into five, one can conclude that two of them are occupied by interference and three of them are occupied by the intended signals. Therefore, $\frac{3}{5}$ is an achievable DOF at the first receiver. Due to symmetry, a similar argument can be applied for the second and third receivers, resulting in $\frac{9}{5}$ as the total DOF of the channel.

\section{Diophantine Approximation: KHINTCHINE-GROSHEV TYPE THEOREMS}

In number theory, the field of Diophantine approximation deals with the approximation of real numbers with rational numbers. The reader is referred to [22], [23] and the references therein. Khintchine theorem is one of the cornerstones in this field. It gives a criteria for a given function $\psi: \mathbb{N} \rightarrow \mathbb{R}_{+}$ and real number $v$ such that $|p+v q|<\psi(|q|)$ has either infinitely many solutions or at most finitely many solutions for $(p, q) \in \mathbb{Z}^{2}$. Let $\mathcal{A}(\psi)$ denote the set of real numbers such 


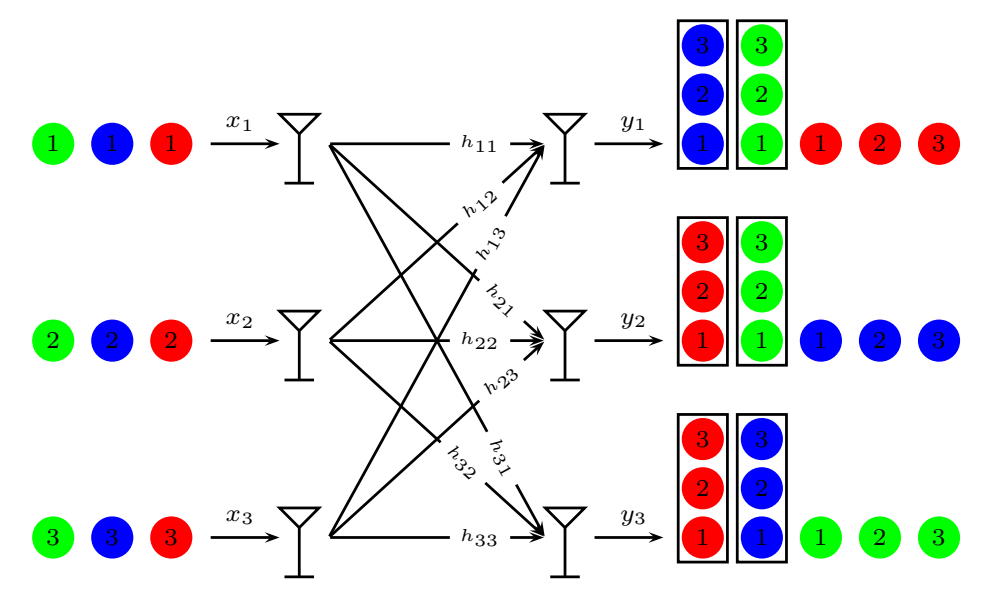

Fig. 6. The $3 \times 3 X$ channel. Each red circle represents a set of data streams intended for the first receiver. They can be aligned with efficiency arbitrary close to one at the second and third receivers, occupying $\frac{1}{5}$ of the received directions. Similarly, signals intended for the second and third receivers can be aligned efficiently at non-intended receivers (blue and green circles). Each set of data stream can carry data with multiplexing gain of $\frac{1}{5}$. There are 9 sets of streams resulting in the total DOF of $\frac{9}{5}$.

that $|p+v q|<\psi(|v|)$ has infinitely many solutions in integers. The theorem has two parts. The first part is the convergence part and states that if $\psi(|q|)$ is convergent, i.e.,

$$
\sum_{q=1}^{\infty} \psi(q)<\infty
$$

then $\mathcal{A}(\psi)$ has measure zero with respect to Lebesque measure. This part can be rephrased in a more convenient way as follows. For almost all real numbers, $|p+v q|>\psi(|q|)$ holds for all $(p, q) \in \mathbb{Z}^{2}$ except for finitely many of them. Since the number of integers violating the inequality is finite, one can find a constant $\kappa$ such that

$$
|p+v q|>\kappa \psi(|q|)
$$

holds for all integers $p$ and $q$ almost surely. The divergence part of the theorem states that $\mathcal{A}(\psi)$ has the full measure, i.e. the set $\mathbb{R}-\mathcal{A}(\psi)$ has measure zero, provided $\psi$ is decreasing and $\psi(|q|)$ is divergent, i.e.,

$$
\sum_{q=1}^{\infty} \psi(q)=\infty
$$

There is an extension to Khintchine's theorem due to Groshev, which regards the rational approximation of linear forms with rational coefficients. Let $\mathbf{v}=\left(v_{1}, v_{2}, \ldots, v_{m}\right)$ and $\mathbf{q}=\left(q_{1}, q_{2}, \ldots, q_{m}\right)$ denote an $m$-tuple in $\mathbb{R}^{m}$ and $\mathbb{Z}^{m}$, respectively. Let $\mathcal{A}_{m}(\psi)$ denote the set of $m$-tuple real numbers $\boldsymbol{g}$ such that

$$
|p+\mathbf{v} \cdot \mathbf{q}|<\psi\left(|\mathbf{q}|_{\infty}\right)
$$

has infinitely many solutions for $p \in \mathbb{Z}$ and $\mathbf{q} \in \mathbb{Z}^{m} .|\mathbf{q}|_{\infty}$ is the supreme norm of $\mathbf{q}$ defined as $\max _{i}\left|q_{i}\right|$. The following theorem gives the Lebesque measure of the set $\mathcal{A}_{m}(\psi)$.

Theorem 4 (Khintchine-Groshev): Let $\psi: \mathbb{N} \rightarrow \mathbb{R}^{+}$. Then the set $\mathcal{A}_{m}(\psi)$ has measure zero, provided

$$
\sum_{q=1}^{\infty} q^{m-1} \psi(q)<\infty
$$

and has the full measure if

$$
\sum_{q=1}^{\infty} q^{m-1} \psi(q)=\infty \quad \text { and } \psi \text { is monotonic. }
$$

In [18], Theorem 4 is used to prove that the total DOF of the two-user $X$ channel can be achieved using a simple coding scheme. It is also proved that the three-user GIC can achieve the DOF of $\frac{4}{3}$ almost surely. Note that Theorem 4 does not include the case where elements of $\mathbf{v}$ are related. It turned out that such a shortcoming in this theorem prevented us from proving the achievablity of $\frac{3}{2}$ for the three-user GIC. Let us assume $\boldsymbol{v}$ lies on a manifold with dimension less than $m$ in $\mathbb{R}^{m}$. In this case, the theorem may not be correct as the measure of the manifold is zero with respect to Lebesque measure. Recently, [24] and [25] independently extended the convergence part of the theorem to the class of non-degenerate manifolds. However, a subclass of non-degenerate manifolds is sufficient for the proofs of the results in this paper. Therefore, in the following theorem we state the theorem in its simplest form by limiting the scope of it.

Theorem 5 ( [24] and [25]): Let $n \leq m, \mathbf{v}=$ $\left(v_{1}, v_{2}, \ldots, v_{n}\right) \in \mathbb{R}^{n}$, and $g_{1}, g_{2}, \ldots, g_{m}$ be functions from $\mathbb{R}^{n}$ to $\mathbb{R}$ with the following conditions:

1) $g_{i}$ for $i \in\{1,2, \ldots, m\}$ is analytic,

2) $1, g_{1}, g_{2}, \ldots, g_{m}$ are linearly independent over $\mathbb{R}$.

For any monotonic function $\psi: \mathbb{N} \rightarrow \mathbb{R}_{+}$such that $\sum_{q=1}^{\infty} q^{m-1} \psi(q)<\infty$ the inequality

$$
\left|p+q_{1} g_{1}(\mathbf{v})+q_{2} g_{2}(\mathbf{v})+\ldots+q_{m} g_{m}(\mathbf{v})\right|<\psi\left(|\mathbf{q}|_{\infty}\right)
$$

has at most finitely many solutions $(p, \mathbf{q}) \in \mathbb{Z} \times \mathbb{Z}^{m}$ for almost all $\mathbf{v} \in \mathbb{R}^{n}$.

Throughout this paper, the function $\psi(q)$ is chosen as $\frac{1}{q^{m+\epsilon}}$ for an arbitrary $\epsilon>0$. Clearly, this function satisfies $(5)$ and is an appropriate candidate for the theorem. If all conditions of the theorem hold, then one can find a constant $\kappa$ such that for almost all $\mathbf{v} \in \mathbb{R}^{n}$

$\left|p+q_{1} g_{1}(\mathbf{v})+q_{2} g_{2}(\mathbf{v})+\ldots+q_{m} g_{m}(\mathbf{v})\right|>\frac{\kappa}{\left(\max _{i}\left|q_{i}\right|\right)^{m+\epsilon}}$ 
holds for all $p \in \mathbb{Z}$ and $\mathbf{q} \in \mathbb{Z}^{m}$.

One class of functions satisfying the conditions in Theorem 5 is of special interest. Let $\mathcal{G}(\mathbf{v})$ denote the set of all monomials with variables from the set $\mathbf{v}=\left\{v_{1}, v_{2}, \ldots, v_{n}\right\}$. In other words, a function $g$ belongs to $\mathcal{G}(\mathbf{v})$ if it can be represented as $g=v_{1}^{s_{1}} v_{2}^{s_{2}} \cdots v_{n}^{s_{m}}$ for some nonnegative integers $s_{1}, s_{2}, \ldots, s_{n}$. It is easy to show that any collection of functions from $\mathcal{G}(\mathbf{v})$ satisfies the conditions of Theorem 5 More specifically, all functions belonging to $\mathcal{G}(\mathbf{v})$ are analytic. Moreover, a set of monomials are independent over $\mathbb{R}$ as long as they are distinct. As a special case when set $\mathbf{v}$ has only one member, i.e. $\mathbf{v}=\{v\}$, then we have $\mathcal{G}(v)=\left\{1, v, v^{2}, v^{3}, \ldots\right\}$.

\section{Coding Scheme And Performance Analysis}

Unless otherwise stated, we assume that the same encoding and decoding schemes are applied at all transmitters and all receivers, respectively. In the following, we will describe the proposed encoding and decoding schemes for a given transmitter and receiver.

Construction of a single data stream: Let us first explain the encoding of a single data stream. The constellation $\mathcal{C}=$ $(-Q, Q)_{\mathbb{Z}}$ as the set of input symbols is chosen. Even though one can use the continuum of real numbers as the input alphabets, restriction to a finite set has the benefit of easy and feasible interference alignment. We assume $Q=\gamma P^{\frac{1-\epsilon}{2(m+\epsilon)}}$ where $\gamma$ is a constant. Notice that since the number of input symbols are bounded by $2 Q-1$, the data stream modulated by $\mathcal{C}$ can at most provide $\frac{1-\epsilon}{m+\epsilon}$ DOF. We will show that at high SNR regimes this DOF can be achieved.

Having formed the constellation, a random codebook with rate $R$ is constructed to change the channel into a reliable one. This can be accomplished by choosing a probability distribution on the input alphabets. The uniform distribution is the first candidate and it is selected for the sake of simplicity. Note that since the constellation is symmetrical by assumption, the expectation of the uniform distribution is zero and the transmit signal has no DC component. The power consumed by the data stream can be loosely upper-bounded as $Q^{2}$.

Remark 2: The parameters involved in the proposed construction, i.e, $Q, m, \gamma$, and $\epsilon$, are universal and applied to all the available data streams. Clearly, the optimum performance of a system can be attained by selecting these parameters appropriately.

Encoding scheme: It is well known that a transmitter with average power constraint $P$ and equipped with $M$ antennas has $M$ degrees-of-freedoms available for data transmission. This is due to the fact that the input signal lies on an $M$-dimensional Euclidean space 1 . As it has been reported in numerous papers, the most applicable approach utilizing these available DOFs relies on the expansion of the input signal into $M$ bases and transmission of a single data stream over each of these bases. For instance, if the input signal is $\mathbf{x} \in \mathbb{R}^{M}$ then by choosing

\footnotetext{
${ }^{1}$ If a channel is time/frequency varying then the input signal over $M$ extensions of time/frequency lies on an $M$-dimensional Euclidean space. Therefore, MIMO and time/frequency varying channels behave the same regarding the DOF.
}

$\left\{\mathbf{T}_{0}, \mathbf{T}_{2}, \ldots, \mathbf{T}_{M-1}\right\}$ independent vectors we have

$$
\mathbf{x}=\sum_{i=0}^{M-1} \mathbf{T}_{i} x_{i},
$$

where $x_{i}$ is the $i$ 'th component of $\mathbf{x}$ in the direction of $\mathbf{T}_{i}$. Being a real number, $x_{i}$ for $i \in\{0,1, \ldots, M-1\}$ can carry at most one DOF. If a transmitter, however, wishes to send less than $M$ data streams, say $L$, then it chooses $L$ bases out of $M$ available bases and discards the rest of bases. In this scheme only integral DOFs are possible for each transmitter. As a simple example, a single antenna transmitter can send a data stream with either one DOF or zero DOF.

In this paper, we prove that the restriction on achieving integral DOFs can be relaxed in a dramatic way. We claim that under some regularity conditions, which are not too restrictive, any fractional DOF is possible. Let us focus on a single antenna transmitter. Viewing as a one-dimensional Euclidean space it has only one base; whereas viewing as a vector field over rational numbers (or equivalently integers), it has infinitely many bases.

The $i$ 'th transmitter chooses a set of real numbers, say $\mathcal{T}_{i}=\left\{T_{i 0}, T_{i 1}, \ldots, T_{i(M-1)}\right\}$, as the set of transmit directions for transmitting $M$ independent data streams. The members of $\mathcal{T}_{i}$ are independent over the field of rational numbers. In the proposed coding scheme, the transmit signal can be represented by

$$
x_{i}=A \sum_{l=0}^{M-1} T_{i l} u_{i l},
$$

where $u_{i l}$ for $l \in\{0,1, \ldots, M-1\}$ is the l'th data stream transmitted in the direction of $T_{i l}$.

The parameter $A$ controls the input power of the transmitters. In what follows, $A$ is computed based on an upper-bound on the input power of a typical transmitter. We start with the following chain of inequalities

$$
\begin{aligned}
E\left[x_{i}^{2}\right] & \stackrel{(a)}{=} A^{2} \sum_{l=0}^{M-1} T_{i l}^{2} E\left[u_{i l}^{2}\right] \\
& \stackrel{(b)}{\leq} A^{2} Q^{2}\left(\sum_{l=0}^{M-1} T_{i l}^{2}\right) \\
& =A^{2} Q^{2} \lambda_{i}^{2}
\end{aligned}
$$

where (a) follows from the fact that all data streams are independent and (b) follows from the fact that the data streams are all the same and hence $u_{i l}^{2} \leq Q^{2}$. We use a short-hand notation $\lambda_{i}$ as $\lambda_{i}=\sum_{l=0}^{M-1} T_{i l}^{2}$. Since each $T_{i l}$ is constant, $\lambda_{i}$ is also a constant. To satisfy the power constraint, it is required that

$$
A \leq \frac{P^{\frac{1}{2}}}{Q \lambda_{i}}
$$

for all $i \in\{1,2, \ldots, K\}$, where $K$ is the number of transmitters. Clearly, it is sufficient to choose

$$
A=\frac{\zeta P^{\frac{1}{2}}}{Q}
$$


where $\zeta=\min _{i} \frac{1}{\lambda_{i}}$. By assumption $Q=\gamma P^{\frac{1-\epsilon}{2(m+\epsilon)}}$. Hence, we have

$$
A=\xi P^{\frac{m-1+2 \epsilon}{2(m+\epsilon)}},
$$

where $\xi=\frac{\zeta}{\gamma}$.

In fact, $A$ and $Q$ are two important design parameters in the encoding. $Q$ controls the cardinality of the input constellation, which in turn provides the maximum achievable rate for individual data streams. The cardinality of the constellation grows roughly as $P^{\frac{1}{2 m}}$. On the other hand, $A$ controls the minimum distance in the received constellation, which in turn affects the performance. Our calculation reveals that no matter how many data streams each transmitter is intended to send, $Q$ and $A$ only depend on $m$, which is the reciprocal of the multiplexing gain of each data streams.

Transmit directions and interference alignment: The most important part of the proposed coding design is the selection of transmit directions. As it is shown in Section III through several examples, transmit directions provide interference alignment as well as separability at all receivers. In fact, to design the optimum directions, interference alignment plays the role as the separability condition usually comes for free.

One important observation is that the transmit directions need to be generated based on channel parameters. Monomials are the best candidates as they are forming a non-degenerate manifold in higher dimensions. This property allows us to incorporate Khintchine-Groshev type theorems in the performance analysis.

We will explain in more details how the transmit directions can be chosen based on the channel coefficients. To have a glimpse on the procedure, we consider there are $K^{\prime}$ receivers in the network receiving signals from $K$ transmitters as interference. The interference, due to all transmitters at Receiver $j$, can be represented as $I_{j}=\sum_{i=1}^{K} h_{j i} x_{i}$ where $h_{j i}$ is a real number. Clearly, if each transmitter transmits one data stream then it is impossible to align them at all receivers. However, it is possible to align the transmit signals if each transmitter encodes $M$ data streams as $x_{i}=\sum_{l=0}^{M-1} T_{i l} u_{i l}$ for all $i \in\{1,2, \ldots, K\}$. Therefore, the interference at the $j$ 'th receiver can be written as

$$
I_{j}=\sum_{i=1}^{K} \sum_{l=0}^{M-1}\left(h_{j i} T_{i l}\right) u_{i l},
$$

where $\left(h_{j i} T_{i l}\right)$ is the received direction for the l'th data stream of the $i$ 'th transmitter. If the transmit directions are chosen randomly, then all of the received directions are distinct and the total number of received directions is $K K^{\prime}$, which is not desirable.

To reduce the number of received directions, $h_{j i}$ 's can be used as the generators for the transmit directions. Let us fix the set of received directions by assuming that all received directions belong to the set $\mathcal{T}_{r}$, which consists of directions of the form $\prod_{i=1}^{K} \prod_{j=1}^{K^{\prime}} h_{j i}^{s_{j i}}$, where $n$ is an arbitrary integer and $0 \leq s_{j i} \leq n$ for all $i \in\{1,2, \ldots, K\}$ and $j \in\left\{1,2, \ldots, K^{\prime}\right\}$. If $M^{\prime}$ denotes the number of received directions then it is easy to show that $M^{\prime}=(n+1)^{K K^{\prime}}$.

A transmit direction is legitimate if it arrives at all receivers with directions belonging to $\mathcal{T}_{r}$. Let us focus on the $i$ 'th transmitter. The received signals due to the transmit signal $x_{i}$ are $h_{1 i} x_{i}, h_{2 i} x_{i}, \ldots, h_{M i} x_{i}$. If we choose the transmit directions from the set $\mathcal{T}_{i}$, which consists of directions of the form

$$
\underbrace{\left(\prod_{j=1}^{K^{\prime}} h_{j i}^{s_{j i}^{\prime}}\right)}_{0 \leq s_{j i}^{\prime} \leq n-1} \underbrace{\left(\prod_{k=1 \& k \neq i}^{K} \prod_{j=1}^{K^{\prime}} h_{j k}^{s_{j k}}\right)}_{0 \leq s_{j k} \leq n},
$$

then the received directions at all receivers belong to $\mathcal{T}_{r}$ as the power of $h_{j i}$ for all $j \in\left\{1,2, \ldots, K^{\prime}\right\}$ is lowered by one. It is easy to show that $M=n^{K^{\prime}}(n+1)^{K^{\prime}(K-1)}$.

The efficiency of the alignment can be measured by the ratio of $M^{\prime}$ and $M$, i.e., $\eta=\frac{M^{\prime}}{M}=\left(\frac{n+1}{n}\right)^{K^{\prime}}$. The perfect alignment happens when $M=M^{\prime}$, i.e., the ratio is one. However, as $n$ can be chosen arbitrarily large, then we can have any efficiency close to one from the proposed alignment technique. In a loose sense, we can say that any number of transmitters can align their signals at any number of receivers.

Decoding scheme: The received signal at the $j$ 'th receiver in its general form can be represented by

$$
y_{j}=A\left(\sum_{l=0}^{L_{j}-1} \bar{T}_{j l} u_{j l}+\sum_{l=0}^{L_{j}^{\prime}-1} \bar{T}_{j l}^{\prime} u_{j l}^{\prime}\right)+z_{j},
$$

where $\bar{T}_{j l}$ and $\bar{T}_{j l}^{\prime}$ are the received directions due to an intended data stream and an interfering signal, respectively. It is assumed that $L_{j}$ and $L_{j}^{\prime}$ are the number of received directions due to the intended data streams and interfering signals, respectively. $u_{j l}$ is an intended data stream. $u_{j l}^{\prime}$ is the an interfering signals. Because of interference alignment, it is possible that $f_{j l}$ data streams arrive at the direction $\bar{T}_{j l}^{\prime}$, which results in $u_{j l}^{\prime} \in\left(-f_{j l} Q, f_{j l} Q\right)_{\mathbb{Z}}$. To have a uniform bound, let us define $f=\max _{(j, l)} f_{j l}$ and $\mathcal{U}^{\prime}=(-f Q, f Q)_{\mathbb{Z}}$. Clearly, $u_{j l}^{\prime} \in \mathcal{U}^{\prime}$ for all $j$ 's and $l$ 's.

We assume that $L_{j}+L_{j}^{\prime} \leq m$ for all $j \in\{1,2, \ldots, K\}$. The $j^{\prime}$ 'th receiver is interested in data streams $u_{j l}$ for all $l \in$ $\left\{0,1, \ldots, L_{j}-1\right\}$. The data stream $u_{j l}$ is decoded as follows. The received signal is first passed through a hard decoder. The hard decoder looks at the received constellation

$$
\mathcal{V}_{j}=A\left(\sum_{l=0}^{L_{j}-1} \bar{T}_{j l} \mathcal{U}+\sum_{l=0}^{L_{j}^{\prime}-1} \bar{T}_{j l}^{\prime} \mathcal{U}^{\prime}\right)
$$

and maps the received signal to the nearest point in the constellation. This changes the continuous channel to a discrete one in which the input symbols are from the transmit constellation $\mathcal{U}$ and the output symbols are from the received constellation $\mathcal{V}_{j}$.

It is assumed that the received constellation has the property that there is a many-to-one map from $\mathcal{V}_{j}$ to $\mathcal{U}_{j}=\sum_{l=0}^{L_{j}-1} \bar{T}_{j l} \mathcal{U}$. Recall that the transmit directions are chosen in such a way that all $u_{j l}$ 's can be recovered uniquely from $\mathcal{U}_{j}$. This, in fact, implies that if there is no additive noise in the channel then the receiver can decode all intended data streams with zero error probability. This property holds, for example, when $\bar{T}_{j l}$ 's and $\bar{T}_{j l}^{\prime}$ are all distinct and linearly independent over 
rational numbers. Throughout this paper, we always design the transmit directions in such a way that this condition holds.

The equivalent channel between $u_{j l}$ and the output of the hard decoder $\hat{u}_{j l}$ becomes a discrete channel and the jointtypical decoder can be used to decode the data stream from a block of $\hat{u}_{j l}$ 's. To decode another data stream, Receiver $j$ performs the same procedure used for decoding $u_{j l}$. In fact, joint-decoding is not used to decode all intended data streams.

Performance Analysis: Let $d_{j_{\min }}$ denote the minimum distance in the received constellation $\mathcal{V}_{j}$. The average error probability in the equivalent discrete channel from input $u_{j l}$ to output $\hat{u}_{j l}$, i.e. $P_{e}=\operatorname{Pr}\left\{\hat{u}_{j l} \neq u_{j l}\right\}$ is bounded as:

$$
P_{e} \leq Q\left(\frac{d_{j_{\min }}}{2}\right) \leq \exp \left(-\frac{d_{j_{\min }}^{2}}{8}\right) .
$$

$P_{e}$ can be used to lower bound the rate achievable for the data stream $u_{j l}$. In [16], Etkin and Ordentlich used Fano's inequality to obtain a lower bound on the achievable rate, which is tight in high SNR regimes. Following similar steps, one can obtain

$$
\begin{aligned}
R_{j l} & =I\left(\hat{u}_{j l}, u_{j l}\right) \\
& =H\left(u_{j l}\right)-H\left(u_{j l} \mid \hat{u}_{j l}\right) \\
& \stackrel{(a)}{\geq} H\left(u_{j l}\right)-1-P_{e} \log |\mathcal{U}| \\
& \stackrel{(b)}{=}\left(1-P_{e}\right) \log |\mathcal{U}|-1 \\
& \stackrel{(c)}{=}\left(1-P_{e}\right) \log (2 Q-1)-1
\end{aligned}
$$

where (a) follows from Fano's inequality, (b) follows from the fact that $u_{j l}$ has a uniform distribution on its range, and (c) follows from the fact that $|\mathcal{U}|$, which is the number of integers in the interval $[-Q, Q]$, is bounded by $2 Q-1$. Let us assume that $P_{e} \rightarrow 0$ as $P \rightarrow \infty$. Under this condition, the achievable multiplexing gain from data stream $u_{j l}$ can be obtained as follows:

$$
\begin{aligned}
r_{j l} & =\lim _{P \rightarrow \infty} \frac{R_{j l}}{0.5 \log P} \\
& \geq \lim _{P \rightarrow \infty} \frac{\log Q}{0.5 \log P} \\
& \stackrel{(a)}{=} \frac{1-\epsilon}{m+\epsilon}
\end{aligned}
$$

where (a) follows from the fact that $Q=\gamma P^{\frac{1-\epsilon}{2(m+\epsilon)}}$. Since $\epsilon>0$ is an arbitrary constant, the multiplexing gain of $\frac{1}{m}$ is achievable for the data stream $u_{j l}$.

Provided that all intended data streams can be successfully decoded at all receivers, the achievable DOF at the $j$ 'th receiver can be written as $\frac{L_{j}}{m}$. However, it is achievable under the condition that $P_{e} \rightarrow 0$ as $P \rightarrow \infty$ and it needs to be shown. To this end, one requires to calculate the minimum distance between points in the received constellation.

Recall that $L_{j}+L_{j}^{\prime} \leq m$ and $\bar{T}_{j l}$ 's and $\bar{T}_{j l}^{\prime}$ 's are all distinct and monomials with variables from the channel coefficients. Theorem 5 can be applied to obtain a lower bound on the minimum distance. Let us assume that one of the directions in $\bar{T}_{j l}$ 's or $\bar{T}_{j l}^{\prime}$, is 1 . Then a point in $\mathcal{V}_{j}$ can be represented as

$$
v=A\left(v_{0}+\sum_{l=1}^{L_{j}+L_{j}-1} \hat{T}_{l} v_{l}\right) .
$$

where $\hat{T}_{l}$ 's are all distinct monomials at receiver $j$. Moreover, $v_{l}$ for all $l \in\left\{0,1, \ldots, L_{j}+L_{j}^{\prime}-1\right\}$ are bounded by $(-f Q, f Q)_{\mathbb{Z}}$. Therefore, the distance between any two points in the received constellation $\mathcal{V}_{j}$ can be bounded using (8) as follows:

$$
d_{j_{\min }}>\frac{\kappa A}{(2 f Q)^{L_{j}+L_{j}^{\prime}-1+\epsilon}} .
$$

Since $L_{j}+L_{j}^{\prime} \leq m$, we have

$$
d_{j_{\min }}>\frac{\kappa A}{(2 f Q)^{m-1+\epsilon}} .
$$

The probability of error in hard decoding (see (13) can be bounded as

$$
P_{e}<\exp \left(-\varrho\left(\frac{A}{Q^{m-1+\epsilon}}\right)^{2}\right),
$$

where $\eta$ is a constant and a function of $\gamma, \kappa, \sigma$, and $\gamma_{i}$ s.

Substituting $A$ and $Q$ in (18) yields

$$
P_{e}<\exp \left(-\eta P^{\epsilon}\right),
$$

which shows that $P_{e}$ has the desired property.

The following theorem summarizes the conditions needed to achieve the multiplexing gain of $\frac{1}{m}$ per data stream.

Theorem 6: Consider there are $K$ transmitters and $K^{\prime}$ receivers in a system parameterized by the channel coefficient vector $\mathbf{h}$. Transmitter $i$ sends $M$ data stream along directions $\mathcal{T}_{i}=\left\{T_{i 0}, T_{i 2}, \ldots, T_{i(M-1)}\right\}$ for all $i \in\{1,2, \ldots, K\}$. The data streams intended for the $j$ 'th receiver arrive at $L_{j}$ directions, which are $\mathcal{T}_{j}=\left\{\bar{T}_{j 0}, \bar{T}_{j 2}, \ldots, \bar{T}_{j\left(L_{j}-1\right)}\right\}$. Moreover, the interference part of the received signal at the $j$ 'th receiver has $L_{j}^{\prime}$ effective data streams with received directions $\mathcal{T}_{j}^{\prime}=$ $\left\{\bar{T}_{j 0}^{\prime}, \bar{T}_{j 2}^{\prime}, \ldots, \bar{T}_{j\left(L_{j}^{\prime}-1\right)}^{\prime}\right\}$ for all $j \in\left\{1,2, \ldots, K^{\prime}\right\}$. Let the following conditions for all $j \in\left\{1,2, \ldots, K^{\prime}\right\}$ hold:

C1 Components of $\mathcal{T}_{i}$ are distinct member of $\mathcal{G}(\mathbf{h})$ and linearly independent over the field of rational numbers.

C2 Components of $\mathcal{T}_{i}$ and $\mathcal{T}_{i}^{\prime}$ are all distinct.

C3 One of the elements of either $\mathcal{T}_{i}$ or $\mathcal{T}_{i}^{\prime}$ is 1 .

Then, by encoding each data stream using the constellation $\mathcal{U}=(-Q, Q)_{\mathbb{Z}}$ where $Q=\gamma P^{\frac{1-\epsilon}{2(m+\epsilon)}}$ and $\gamma$ is a constant, the following DOF is achievable for almost all realizations of the system:

$$
r_{\text {sum }}=\frac{L_{1}+L_{2}+\cdots+L_{K^{\prime}}}{m},
$$

where $m$ is the maximum received directions among all receivers, i.e., $m=\max _{i} L_{i}+L_{i}^{\prime}$.

Remark 3: If $\mathrm{C} 2$ holds, then the measure of the event "components of $\mathcal{T}_{i}$ and $\mathcal{T}_{i}^{\prime}$ are dependent over the field of rational numbers" is zero.

Remark 4: If C3 does not hold, then by adding a virtual data stream in the direction 1 at the receiver, one can conclude that $\frac{1}{m+1}$ is achievable for all data streams. 


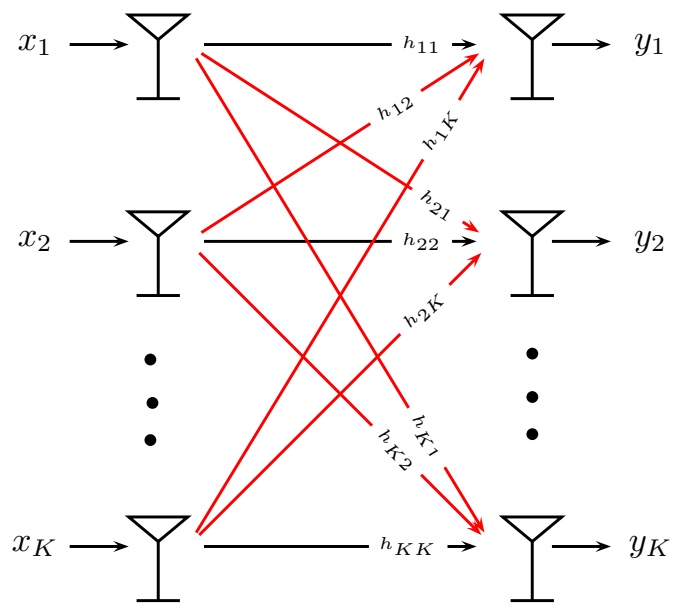

Fig. 7. The $K$-user GIC. User $i$ for $i \in\{1,2, \ldots, K\}$ wishes to communicate with its corresponding receiver while receiving interference from other users.

Theorem 6 implies that the most difficult part of the design is the selection of transmit directions for all users. This is due to the fact that random selection results in $m=\sum_{i=1}^{K} L_{i}$ received directions, which in turn provides $1 \mathrm{DOF}$ for the channel. A careful design is needed to reduce the number of received directions at all users. In the following section, we provide such a design for the $K$-user GIC.

\section{VI. $K$-USER Gaussian INTERfEREnCE ChanNEL}

\section{A. System Model}

The $K$-user GIC models a network in which $K$ transmitterreceiver pairs (users) sharing a common bandwidth wish to have reliable communication at their maximum rates. The channel's input-output relation can be stated as follows, see Figure 7

$$
\begin{aligned}
& y_{1}=h_{11} x_{1}+h_{12} x_{2}+\ldots+h_{1 K} x_{K}+z_{1}, \\
& y_{2}=h_{21} x_{1}+h_{22} x_{2}+\ldots+h_{2 K} x_{K}+z_{2} \text {, } \\
& \vdots=\quad \vdots \quad \vdots \quad \ddots \quad \vdots \\
& y_{K}=h_{K 1} x_{1}+h_{K 2} x_{2}+\ldots+h_{K K} x_{K}+z_{K} \text {, }
\end{aligned}
$$

where $x_{i}$ and $y_{i}$ are input and output symbols of User $i$ for $i \in\{1,2, \ldots, K\}$, respectively. $z_{i}$ is Additive White Gaussian Noise (AWGN) with unit variance for $i \in\{1,2, \ldots, K\}$. Transmitters are subject to the power constraint $P . h_{j i}$ represents the channel gain between Transmitter $i$ and Receiver $j$. It is assumed that all channel gains are real and time invariant. The set of all channel gains is denoted by $\mathbf{h}$, i.e., $\mathbf{h}=\left\{h_{11}, \ldots, h_{1 K}, h_{21}, \ldots, h_{2 K}, \ldots, h_{K 1}, \ldots, h_{K K}\right\}$. Since the noise variances are normalized, the Signal to Noise Ratio (SNR) is equivalent to the input power $P$. Hence, we use them interchangeably throughout this paper.

In this paper, we are primarily interested in characterizing the total DOF of the $K$-user GIC. Let $\mathcal{C}$ denote the capacity region of this channel. The DOF region associated with the channel is in fact the shape of $\mathcal{C}$ in high SNR regimes scaled by $\log$ SNR. Let us denote the DOF region by $\mathcal{R}$. All extreme points of $\mathcal{R}$ can be identified by solving the following optimization problem:

$$
r_{\boldsymbol{\lambda}}=\lim _{\mathrm{SNR} \rightarrow \infty} \max _{\mathbf{R} \in \mathcal{C}} \frac{\boldsymbol{\lambda}^{t} \mathbf{R}}{0.5 \log \mathrm{SNR}} .
$$

The total DOF refers to the case where $\boldsymbol{\lambda}=\{1,1, \ldots, 1\}$, i.e., the sum-rate is concerned. Throughout this paper, $r_{\text {sum }}$ denotes the total DOF of the system.

An upper bound on the DOF of this channel is obtained in [10]. The upper bound states that the total DOF of the channel is less than $\frac{K}{2}$, which means each user can at most enjoy one half of its maximum DOF.

\section{B. Three-user Gaussian Interference Channel: DOF $=\frac{3}{2}$ is Achievable}

In this section, we consider the three-user GIC and explain in detail that, by an appropriate selection of transmit directions, the DOF of $\frac{3}{2}$ is achievable for almost all cases. We will explain in more detail that by an appropriate selection of transmit directions this DOF can be achieved.

In [18], we defined the standard model of the three-user GIC. The definition is as follows:

Definition 1: The three user interference channel is called standard if it can be represented as

$$
\begin{aligned}
& y_{1}=G_{1} x_{1}+x_{2}+x_{3}+z_{1} \\
& y_{2}=G_{2} x_{2}+x_{1}+x_{3}+z_{2} \\
& y_{3}=G_{3} x_{3}+x_{1}+G_{0} x_{2}+z_{3} .
\end{aligned}
$$

where $x_{i}$ for User $i$ is subject to the power constraint $P . z_{i}$ at Receiver $i$ is AWGN with unit variance.

In [18], it is also proved that every three-user GIC has an equivalent standard channel as far as the DOF is concerned. The parameters in the standard channel are related to the parameters of the original one thorough the following equations.

$$
\begin{aligned}
G_{0} & =\frac{h_{13} h_{21} h_{32}}{h_{12} h_{23} h_{31}}, \\
G_{1} & =\frac{h_{11} h_{12} h_{23}}{h_{12} h_{21} h_{13}}, \\
G_{2} & =\frac{h_{22} h_{13}}{h_{12} h_{23}}, \\
G_{3} & =\frac{h_{33} h_{12} h_{21}}{h_{12} h_{23} h_{31}} .
\end{aligned}
$$

As mentioned in the previous section, transmit directions are monomials with variables from channel coefficients. For the three user case, we only use $G_{O}$ as the generator of transmit directions. Therefore, transmit directions are selected from the set $\mathcal{G}\left(G_{0}\right)$, which is a subset of $\mathcal{G}\left(G_{0}, G_{1}, G_{2}, G_{3}\right)$. Clearly, $\mathcal{G}\left(G_{0}\right)=\left\{1, G_{0}, G_{0}^{2}, G_{0}^{3}, \cdots\right\}$.

We consider two different cases based on the value of $G_{0}$ being algebraic or transcendental. Although the measure of being algebraic is zero, we prove that for each case the total DOF can be achieved if the transmit and receive directions satisfy the conditions of Theorem 6 We start with the case where $G_{0}$ is algebraic. 
1) Case I: $G_{0}$ is algebraic: By definition, if $G_{0}$ is algebraic then it is a root of a polynomial with integer coefficients. Let us assume $G_{0}$ satisfies

$$
a_{d} G_{0}^{d}+a_{d-1} G_{0}^{d-1}+\ldots+a_{1} G_{0}+a_{0}=0,
$$

where $a_{d}, a_{d-1}, \ldots, a_{0}$ are integers. In other words, the set $\mathcal{T}=\left\{1, G_{0}, G_{0}^{2}, \ldots, G_{0}^{d-1}\right\}$ is a basis for $\mathcal{G}\left(G_{0}\right)$ over rational numbers. Therefore, as the transmit directions need to be independent over the field of rational numbers, the transmitters are restricted to choose their transmit directions among numbers in $\mathcal{T}$. We assume that all transmitters transmit along all directions in $\mathcal{T}$, i.e., $\mathcal{T}_{i}=\mathcal{T}$ for all $i \in\{1,2,3\}$. By this selection, $\mathrm{C} 1$ in Theorem 6 holds for all transmitters.

In this case, Transmitter $i$ sends $L_{i}=d$ data streams as follows

$$
x_{i}=A \sum_{j=0}^{d-1} G_{0}^{j} u_{i j},
$$

for all $i \in\{1,2,3\}$. The received signal at Receiver 1 can be written as

$$
y_{1}=A\left(\sum_{j=0}^{d-1} G_{1} G_{0}^{j} u_{1 j}+\sum_{j=0}^{d-1} G_{0}^{j} u_{1 j}^{\prime}\right)+z_{1}
$$

where $u_{1 j}^{\prime}=u_{2 j}+u_{3 j}$ for all $j \in\{0,1, \ldots, d-1\}$. The signals from Transmitters 2 and 3 are aligned and the number of received directions is $L_{1}^{\prime}=d$. Moreover $\mathrm{C} 2$ and $\mathrm{C} 3$ in Theorem 6 hold for this receiver. Since the received signal at Receiver 2 is similar to that of Receiver 1, we can deduce that $L_{2}^{\prime}=d$ and C2 and C3 hold.

The received signal at Receiver 3 can be written as

$$
y_{3}=A\left(\sum_{j=0}^{d-1} G_{3} G_{0}^{j} u_{3 j}+\sum_{j=0}^{d} G_{0}^{j} u_{3 j}^{\prime}\right)+z_{3}
$$

where $u_{3 j}^{\prime}=u_{2 j}+u_{1(j-1)}$ for $j \in\{1,2, \ldots, d-1\}, u_{30}^{\prime}=$ $u_{20}$, and $u_{3 d}^{\prime}=u_{1 d}$. The number of received directions from interfering users is $d+1$. However, they are not independent over the field of rational numbers. Using (24), $G_{0}^{d}$ can be represented as a linear combination of $\left\{1, G_{0}, G_{0}^{2}, \ldots, G_{0}^{d-1}\right\}$ with rational coefficients. Multiplying both sides of 27 by $a_{d}$, we have

$\tilde{y}_{3}=A\left(\sum_{j=0}^{d-1} a_{d} G_{3} G_{0}^{j} u_{3 j}+\sum_{j=0}^{d-1} G_{0}^{j} a_{d} u_{3 j}^{\prime}+a_{d} G_{0}^{d} u_{3 d}^{\prime}\right)+\tilde{z}_{3}$,

where $\tilde{y}_{3}=a_{d} y_{3}$ and $\tilde{z}_{3}=a_{d} z_{3}$. Substituting from (27), we obtain

$$
\tilde{y}_{3}=A\left(\sum_{j=0}^{d-1} a_{d} G_{3} G_{0}^{j} u_{3 j}+\sum_{j=0}^{d-1} G_{0}^{j}(\underbrace{a_{d} u_{3 j}^{\prime}-a_{j} u_{3 d}^{\prime}}_{u_{j}^{\prime \prime}})\right)+\tilde{z}_{3} .
$$

Clearly, $L_{3}^{\prime}=d$ and C2 and C3 hold for this receiver as well.

The maximum number of received directions at all receivers is $m=2 d$. Since $\mathrm{C} 1, \mathrm{C} 2$, and $\mathrm{C} 3$ hold at all receivers, by applying Theorem 6 we conclude that the total DOF of $\frac{3}{2}$ is achievable for almost all cases.
Remark 5: In a special case, $d=1$ in (24). In other words, $G_{0}$ is a rational number. This case is considered in [16] and it is proved that it can achieve the total DOF of the channel.

2) Case II: $G_{0}$ is transcendental: If $G_{0}$ is transcendental then all members of $\mathcal{G}\left(G_{0}\right)$ are linearly independent over the field of rational numbers. Hence, we are not limited to any subset of $\mathcal{G}\left(G_{0}\right)$, as far as the independence of transmit directions is concerned. We will show that $\frac{3 n+1}{2 n+1}$ is an achievable DOF for any $n \in \mathbb{N}$. To this end, we propose a design that is not symmetrical.

Transmitter 1 uses the set of directions $\mathcal{T}_{1}=$ $\left\{1, G_{0}, G_{0}^{2}, \ldots, G_{0}^{n}\right\}$ to transmit $L_{1}=n+1$ to its corresponding receiver. Clearly $\mathcal{T}_{1}$ satisfies $\mathrm{C} 1$. The transmit signal from User 1 can be written as

$$
x_{1}=A \sum_{j=0}^{n} G_{0}^{j} u_{1 j} .
$$

Transmitters 2 and 3 transmit in $L_{2}=L_{3}=n$ directions using $\mathcal{T}_{2}=\mathcal{T}_{3}=\left\{1, G_{0}, G_{0}^{2}, \ldots, G_{0}^{n-1}\right\}$. Clearly both $\mathcal{T}_{2}$ and $\mathcal{T}_{3}$ satisfy $\mathrm{C} 1$. The transmit signals can be expressed as

$$
x_{2}=A \sum_{j=0}^{n-1} G_{0}^{j} u_{2 j}
$$

and

$$
x_{3}=A \sum_{j=0}^{n-1} G_{0}^{j} u_{3 j} .
$$

The received signal at Receiver 1 can be expressed as:

$$
y_{1}=A\left(\sum_{j=0}^{n} G_{1} G_{0}^{j} u_{1 j}+\sum_{j=0}^{n-1} G_{0}^{j} u_{1 j}^{\prime}\right)+z_{1},
$$

where $u_{1 j}^{\prime}=u_{2 j}+u_{3 j}$. In fact, transmit signals from Users 2 and 3 are aligned at Receiver 1 . This is due to the fact that out of $2 n$ possible received directions, only $n$ directions are effective, i.e., $L_{1}^{\prime}=n$. One can also confirm that $\mathrm{C} 2$ and $\mathrm{C} 3$ hold at Receiver 1.

The received signal at Receiver 2 can be expressed as:

$$
y_{2}=A\left(\sum_{j=0}^{n-1} G_{2} G_{0}^{j} u_{2 j}+\sum_{j=0}^{n} G_{0}^{j} u_{2 j}^{\prime}\right)+z_{2}
$$

where $u_{2 j}^{\prime}=u_{1 j}+u_{3 j}$ for all $j \in\{0,1, \ldots, n-1\}$ and $u_{2 n}^{\prime}=$ $u_{1 n}$. At Receiver 2, transmitted signals from Users 1 and 3 are aligned and the number of effective received directions is $L_{2}^{\prime}=n+1$. Moreover, it can be easily seen that C2 and C3 hold at Receiver 2.

The received signal at Receiver 3 can be expressed as:

$$
y_{3}=A\left(\sum_{j=0}^{n-1} G_{3} G_{0}^{j} u_{3 j}+\sum_{j=0}^{n} G_{0}^{j} u_{3 j}^{\prime}\right)+z_{3},
$$

where $u_{3 j}^{\prime}=u_{1 j}+u_{2 j}$ for all $j \in\{1,2, \ldots, n\}$ and $u_{30}^{\prime}=$ $u_{10}$. At Receiver 3, transmitted signals from Users 1 and 2 are aligned and the number of effective received directions is $L_{2}^{\prime}=n+1$. Clearly, C2 and C3 hold for Receiver 3. 
Since C1, C2, and C3 hold at all users, we only need to obtain the number of maximum received directions at all receivers. To this end, we observe that

$$
m=\max \left\{L_{1}+L_{1}^{\prime}, L_{2}+L_{2}^{\prime}, L_{3}+L_{3}^{\prime}\right\}=2 n+1
$$

. Therefore, an application of Theorem 6 reveals that the following DOF is achievable.

$$
\begin{aligned}
r_{\text {sum }} & =\frac{L_{1}+L_{2}+L_{3}}{m} \\
& =\frac{3 n+1}{2 n+1} .
\end{aligned}
$$

Since $n$ is an arbitrary integer, one can conclude that $\frac{3}{2}$ is achievable for the three-user GIC almost surely.

\section{K-user Gaussian Interference Channel: DOF $=\frac{K}{2}$ is Achievable}

In this section, we prove the main theorem of this paper, i.e., the DOF of $\frac{K}{2}$ is achievable for the $K$-user GIC. As pointed out in Section $\mathrm{V}$, we need to design the transit directions of all transmitters in such a way that they satisfy the conditions of Theorem 6. Recall that all transmit directions are monomials with variables in $\mathbf{h}$. We reserve the direct gains and do not use them as generating variables. The reason is that $\mathrm{C} 2$ in Theorem 6 requires that all received directions be distinct. By setting aside the direct gains, a transmit direction from the intended user is multiplied by the direct gain and therefore it is distinct from all other transmit directions (by $\mathrm{C} 1$ all transmit directions from a user are distinct).

We assume that all channel gains are transcendental. On the one hand, since the measure of being algebraic is zero, this assumption is innocuous. On the other hand, as we learned from the three-user case algebraic gains are beneficial as they reduce the number of transmit directions required to achieve the total DOF of the channel.

We start with selecting the transmit directions for User $i$. A direction $T \in \mathcal{G}(\mathbf{h})$ is chosen as the transmit direction for User $i$ if it can be represented as

$$
T=\prod_{j=1}^{K} \prod_{l=1}^{K} h_{j l}^{s_{j l}}
$$

where $s_{j l}$ 's are integers satisfying

$$
\begin{cases}s_{j j}=0 & \forall j \in\{1,2, \ldots, K\} \\ 0 \leq s_{j i} \leq n-1 & \forall j \in\{1,2, \ldots, K\} \& j \neq i \\ 0 \leq s_{j l} \leq n & \text { Otherwise. }\end{cases}
$$

The set of all transmit directions is denoted by $\mathcal{T}_{i}$. It is easy to show that the cardinality of this set is

$$
L_{i}=n^{K-1}(n+1)^{(K-1)^{2}} .
$$

Clearly, $\mathcal{T}_{i}$ satisfies $\mathrm{C} 1$ for all $i \in\{1,2, \ldots, K\}$.

To compute $L_{i}^{\prime}$ (the number of independent received directions due to interference), we investigate the effect of Transmitter $k$ on Receiver $i$. Let us first define $\mathcal{T}_{r}$ as the set of directions represented by (34) and satisfying

$$
\begin{cases}s_{j j}=0 & \forall j \in\{1,2, \ldots, K\} \\ 0 \leq s_{j l} \leq n & \text { Otherwise. }\end{cases}
$$

We claim that $\mathcal{T}_{i k}$, the set of received directions at Receiver $i$ due to Transmitter $k$, is a subset of $\mathcal{T}_{r}$. In fact, all transmit directions of Transmitter $k$ arrive at Receiver $i$ multiplied by $h_{i k}$. Based on the selection of transmit directions, however, the maximum power of $h_{i k}$ in all members of $\mathcal{T}_{i k}$ is $n-1$. Therefore, none of the received directions violates the condition of (46) and this proves the claim.

Since $\mathcal{T}_{r}$ is not related to User $k$, one can conclude that $\mathcal{T}_{i k} \subseteq \mathcal{T}_{r}$ for all $k \in\{1,2, \ldots, K\}$ and $k \neq i$. Hence, we deduce that all interfering users are aligned in the directions of $\mathcal{T}_{r}$. Now, $L_{i}^{\prime}$ can be obtained by counting the members of $\mathcal{T}_{r}$. It is easy to show that

$$
L_{i}^{\prime}=(n+1)^{K(K-1)} \text {. }
$$

The received directions at Receiver $i$ are members of $h_{i i} \mathcal{T}_{i}$ and $\mathcal{T}_{r}$. Since $h_{i i}$ does not appear in members of $\mathcal{T}_{r}$, the members of $h_{i i} \mathcal{T}_{i}$ and $\mathcal{T}_{r}$ are distinct. Therefore, C2 holds at Receiver $i$. Since all the received directions are irrationals, C3 does not hold at Receiver $i$.

Since $C_{1}$ and $C_{2}$ hold for all users, we can apply Theorem 6 to obtain the DOF of the channel. We have

$$
\begin{aligned}
r_{\text {sum }} & =\frac{L_{1}+L_{2}+\ldots+L_{K}}{m+1} \\
& =\frac{K n^{K-1}(n+1)^{(K-1)^{2}}}{m+1}
\end{aligned}
$$

where $m$ is

$$
\begin{aligned}
m & =\max _{i} L_{i}+L_{i}^{\prime} \\
& =n^{K-1}(n+1)^{(K-1)^{2}}+(n+1)^{K(K-1)} .
\end{aligned}
$$

Combining the two equations, we obtain

$$
r_{\text {sum }}=\frac{K}{1+\left(\frac{n+1}{n}\right)^{K-1}+\frac{1}{n^{K-1}(n+1)^{(K-1)^{2}}}} .
$$

Since $n$ can be arbitrary large, we conclude that $\frac{K}{2}$ is achievable for the $K$-user GIC.

\section{Cellular Systems: Uplink}

\section{A. System Model}

In a cellular network, an area is partitioned into several cells and, within each cell, there is a base station serving users inside the cell. There are two modes of operation. In the uplink mode, users within a cell transmit independent messages to the base station in the cell; whereas in the downlink mode, the base station broadcasts independent messages to all users inside the cell. In this section, we only consider the uplink mode. Abstractly, the uplink mode corresponds to a network in which several Multiple Access Channels (MAC) share the same spectrum for data transmission. Let us assume there exist $M$ users in each MAC and there are $K$ MACs in the network. The received signal at the base station in Cell $k$ can be represented as

$$
y_{k}=\underbrace{\sum_{l=1}^{M} h_{k(k l)} x_{k l}}_{\text {users within the cell }}+\underbrace{\sum_{i=1 \& i \neq k}^{K} I_{k i}}_{\text {intra cell interference }}+z_{k}
$$


where $I_{k i}$ is the aggregate interference from all users in Cell $i$, i.e.,

$$
I_{k i}=\sum_{l=1}^{M} h_{k(i l)} x_{i l} .
$$

Let $\mathcal{C}_{\text {up }}$ denote the capacity region of this channel. The DOF region associated with the channel can be defined as the shape of the region in high SNR regimes scaled by $\log$ SNR. Let us denote the DOF region by $\mathcal{R}_{\text {up }}$. We are primarily interested in the main facet of the DOF region defined as:

$$
r_{\text {up }}=\lim _{\mathrm{SNR} \rightarrow \infty} \max _{\mathbf{R} \in \mathcal{C}_{\text {up }}} \frac{\sum_{k=1}^{K} \sum_{l=1}^{M} R_{k l}}{0.5 \log \mathrm{SNR}},
$$

where $R_{k l}$ is an achievable rate for the l'th user in Cell $k$.

\section{B. The Total DOF of $\frac{K M}{M+1}$ is Achievable}

To obtain an upper bound on the total DOF of this channel, we assume that all users within a cell can cooperate. This cooperation converts the uplink mode to a MISO $K$-user GIC with $M$ antennas at the transmitters and one antenna at the receivers. An upper bound on the DOF of the MISO $K$-user GIC is obtained in [20]. The upper bound states that the total DOF of the channel is less than $\frac{K M}{M+1}$. We will show that this DOF is achievable.

We start with selecting the transmit directions of the $m$ 'th user in Cell $k$. A direction $T \in \mathcal{G}(\mathbf{H})(\mathbf{H}$ is the set of all channel gains) is chosen as the transmit direction for this user if it can be represented as

$$
T=\prod_{j=1}^{K} \prod_{i=1}^{K} \prod_{l=1}^{M} h_{j(i l)}^{s_{j(i l)}},
$$

where $s_{j(i l)}$ 's are integers satisfying

$$
\begin{cases}s_{j(j l)}=0 & \forall j \in\{1,2, \ldots, K\} \& l \in\{1, \ldots, M\} \\ 0 \leq s_{j(k m)} \leq n-1 & \forall j \in\{1,2, \ldots, K\} \& j \neq k \\ 0 \leq s_{j(i l)} \leq n & \text { Otherwise. }\end{cases}
$$

The set of all transmit directions is denoted by $\mathcal{T}_{k m}$. It is easy to show that the cardinality of this set is

$$
L_{k m}=n^{K-1}(n+1)^{(K M-1)(K-1)} .
$$

Clearly, $\mathcal{T}_{k m}$ satisfies $\mathrm{C} 1$.

We claim that all signals from non-intended cells are aligned at all base stations. In order to prove the claim, we introduce $\mathcal{T}_{i}$ as the set of received direction due to interference at the $i$ 'th base stations. Clearly,

$$
\mathcal{T}_{i}=\bigcup_{k=1 \& k \neq i}^{K} \bigcup_{m=1}^{M}\left(h_{i(k m)} \mathcal{T}_{k m}\right) .
$$

Let us define $\mathcal{T}$ as the set of directions represented by (44) and satisfying

$$
\begin{cases}s_{j(j l)}=0 & \forall j \in\{1,2, \ldots, K\} \& l \in\{1,2, \ldots, M\} \\ 0 \leq s_{j(i l)} \leq n & \text { Otherwise. }\end{cases}
$$

We claim that $\mathcal{T}_{i} \subseteq \mathcal{T}$. In fact, all transmit directions of the $m^{\prime}$ th user in Cell $k$ arrive at Receiver $i$ multiplied by

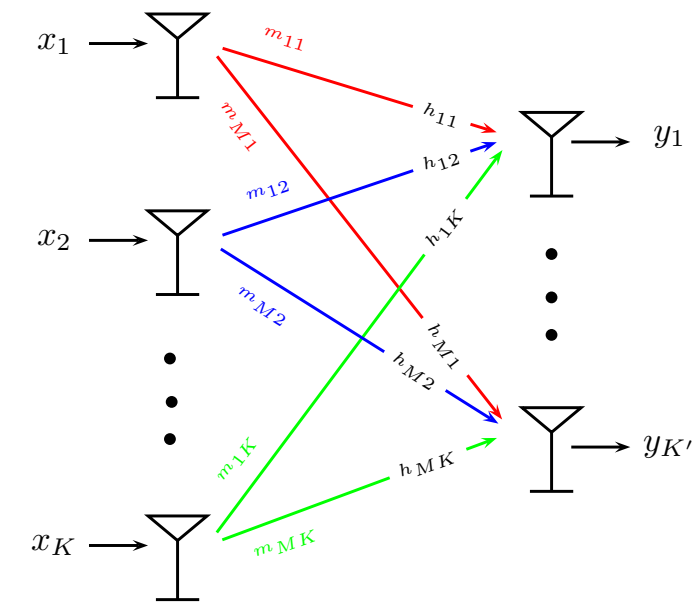

Fig. 8. The $K \times M X$ Channel. User $i$ for $i \in\{1,2, \ldots, K\}$ wishes to transmit an independent message $m_{j i}$ to Receiver $j$ for all $j \in\{1,2, \ldots, M\}$.

$h_{i(\mathrm{~km})}$. Based on the selection of transmit directions, however, the maximum power of $h_{i(\mathrm{~km})}$ in all members of $\mathcal{T}_{k m}$ is $n-1$. Therefore, none of the received directions violates the condition (46) and this proves the claim.

Since $\mathcal{T}$ is not related to the $i$ 's base station, one can conclude that $\mathcal{T}_{i} \subseteq \mathcal{T}$ for all $i \in\{1,2, \ldots, K\}$. Hence, we deduce that all interfering users are aligned in the directions of $\mathcal{T}$. Now, $L_{i}^{\prime}$ can be obtained by counting the members of $\mathcal{T}_{r}$. It is easy to show that

$$
L_{i}^{\prime}=(n+1)^{M K(K-1)} .
$$

The total number of received directions at the $i$ 'th base stations is $\sum_{l=1}^{M} L_{i l}+L_{i}^{\prime}$. Since $C_{1}$ and $C_{2}$ hold at all base stations, we can obtain the total DOF of the channel as

$$
\begin{aligned}
r_{\text {sum }} & =\frac{\sum_{k=1}^{K} \sum_{m=1}^{M} L_{k m}}{M n^{K-1}(n+1)^{(K M-1)(K-1)}+(n+1)^{M K(K-1)}+1} \\
& =\frac{M K n^{K-1}(n+1)^{(K M-1)(K-1)}}{M n^{K-1}(n+1)^{(K M-1)(K-1)}+(n+1)^{M K(K-1)}+1} \\
& =\frac{M K}{M+\left(\frac{n+1}{n}\right)^{K-1}+\frac{1}{n^{K-1}(n+1)^{(K M-1)(K-1)}}}
\end{aligned}
$$

Since $n$ can be arbitrary large, we conclude that $\frac{M K}{M+1}$ is achievable for the uplink of a cellular system.

\section{VIII. $K \times M X$ CHANNEL}

\section{A. System Model}

The $K \times M X$ channel models a network in which $K$ transmitters wish to communicate with $M$ receivers. Unlike the interference channel, each transmitter has a message for each receiver. In other words, Transmitter $i$ for all $i \in\{1,2, \ldots, K\}$ wishes to transmit an independent message to Receiver $j$ for all $j \in\{1,2, \ldots, M\}$. The message transmitted by Transmitter $i$ and intended for Receiver $j$ is denoted by $m_{j i}$. The channel's 
input-output relation can be stated as follows, see Figure 8 ,

$$
\begin{aligned}
& y_{1}=h_{11} x_{1}+h_{12} x_{2}+\ldots+h_{1 K} x_{K}+z_{1}, \\
& y_{2}=h_{21} x_{1}+h_{22} x_{2}+\ldots+h_{2 K} x_{K}+z_{2} \text {, } \\
& \vdots=\quad \vdots \quad \vdots \quad \ddots \quad \vdots \\
& y_{M}=h_{M 1} x_{1}+h_{M 2} x_{2}+\ldots+h_{M K} x_{K}+z_{M} \text {, }
\end{aligned}
$$

where $x_{i}$ and $y_{i}$ are input and output symbols of User $i$ for $i \in\{1,2, \ldots, K\}$, respectively. $z_{i}$ is Additive White Gaussian Noise (AWGN) with unit variance for $i \in\{1,2, \ldots, K\}$. Transmitters are subject to the power constraint $P . h_{j i}$ represents the channel gain between Transmitter $i$ and Receiver $j$. It is assumed that all channel gains are real and time invariant.

Let $\mathcal{C}_{X}$ denote the capacity region of this channel. The DOF region associated with the channel can be defined as the shape of the region in high SNR regimes scaled by $\log$ SNR. Let us denote the DOF region by $\mathcal{R}_{X}$. We are primarily interested in the main facet of the DOF region defined as:

$$
r_{\mathrm{Xsum}}=\lim _{\mathrm{SNR} \rightarrow \infty} \max _{\mathbf{R} \in \mathcal{C}_{X}} \frac{\sum_{i=1}^{K} \sum_{j=1}^{M} R_{i j}}{0.5 \log \mathrm{SNR}},
$$

where $R_{i j}$ is an achievable rate for the message $m_{i j}$ and $\mathbf{R}$ is the set of all achievable rates. The DOF achievable by the message $m_{i j}$ is denoted by $r_{i j}$.

\section{B. The Total DOF of $\frac{K M}{K+M-1}$ is Achievable}

An upper bound on the DOF of this channel is obtained in [11]. The upper bound states that the total DOF of the channel is less than $\frac{K M}{K+M-1}$, which means each message can at most achieve $\frac{1}{K+M-1}$ of DOF. We will show that this DOF is achievable. To this end, Transmitter $i$ for all $i \in\{1,2, \ldots, K\}$ transmits $M$ signals along $M$ directions as follows:

$$
x_{i}=\sum_{j=1}^{M} h_{j i} x_{j i}
$$

where $x_{j i}$ is the signal carrying the message $m_{j i}$. Let us focus on the signals intended for Receiver 1 , i.e., $x_{11}, x_{12}, \ldots, x_{1 K}$. The received signals due to these transmit signals can be written as

$$
\begin{aligned}
& \tilde{y}_{1}=h_{11}^{2} x_{11}+h_{12}^{2} x_{12}+\ldots+h_{1 K}^{2} x_{1 K} \\
& I_{21}=\left(h_{21} h_{11}\right) x_{11}+\left(h_{22} h_{12}\right) x_{12}+\ldots+\left(h_{2 K} h_{1 K}\right) x_{1 K} \\
& \vdots=\quad \vdots \quad \vdots \quad \cdots \quad \vdots \\
& I_{M 1}=\left(h_{M 1} h_{11}\right) x_{11}+\left(h_{M 2} h_{12}\right) x_{12}+\ldots+\left(h_{M 1} h_{1 K}\right) x_{1 K} \text {. }
\end{aligned}
$$

Since $x_{11}, x_{12}, \ldots, x_{1 K}$ are not intended for Receiver $j$ for all $j \in\{2,3, \ldots, M\}, I_{j 1}$ is a part of interference at Receiver $j$. We claim that we can align all interfering signals $x_{11}, x_{12}, \ldots, x_{1 K}$ at all Receivers $j \in\{2,3, \ldots, M\}$.

Let $\mathbf{H}_{1}$ denote the set of all coefficients appeared in $I_{21}, I_{31}, \ldots, I_{M 1}$, i.e., $\mathbf{H}_{1}=\left\{\left(h_{21} h_{11}\right)\right.$, $\left.\left.\left(h_{22} h_{12}\right), \ldots,\left(h_{M 2} h_{12}\right), h_{M 1} h_{1 K}\right)\right\} . \quad \mathbf{H}_{1}$ has $(M-1) K$ members. The set of all monomials with variables in $\mathbf{H}_{1}$ is denoted by $\mathcal{G}\left(\mathbf{H}_{1}\right)$. Let $\mathcal{T}_{1}$ denote a subset of $\mathcal{G}\left(\mathbf{H}_{1}\right)$ consisting of monomials represented by

$$
T=\prod_{i=1}^{K} \prod_{j=1}^{M}\left(h_{j i} h_{1 i}\right)^{s_{j i}}
$$

where

$$
\begin{cases}s_{1 i}=0 & \forall i \in\{1,2, \ldots, K\} \\ 0 \leq s_{j i} \leq n & \text { Otherwise. }\end{cases}
$$

Clearly, $\mathcal{T}_{1}$ has $(n+1)^{(M-1) K}$ members.

The message $m_{1 i}$ for $i \in\{1,2, \ldots, K\}$ is transmitted along directions in $\mathcal{T}_{1 i}$ where $\mathcal{T}_{1 i} \subset \mathcal{T}_{1}$. A direction $T$ in $\mathcal{T}_{1 i}$ can be represented as

$$
T=\prod_{l=1}^{K} \prod_{j=1}^{M}\left(h_{j l} h_{1 l}\right)^{s_{j l}}
$$

where

$$
\begin{cases}s_{1 l}=0 & \forall l \in\{1,2, \ldots, K\} \\ 0 \leq s_{j i} \leq n-1 & \forall j \in\{1,2, \ldots, M\} \& j \neq 1 \\ 0 \leq s_{j l} \leq n & \text { Otherwise. }\end{cases}
$$

It is easy to show that the cardinality of $\mathcal{T}_{1 i}$ is $n^{M-1}(n+$ $1)^{(M-1)(K-1)}$. The received directions due to $x_{1 i}$ at all receivers belong to $\mathcal{T}_{1}$. In fact, $x_{1 i}$ arrives at receiver $j$ multiplied by $\left(h_{j i} h_{1 i}\right)$ and since the power of $\left(h_{j i} h_{1 i}\right)$ in all directions in $x_{1 i}$ is less than $n$ we conclude that the received directions are all in $\mathcal{T}_{1}$. Therefore, all transmit signals are aligned and the total number of directions in $I_{j 1}$ for all $j \in\{2,3, \ldots, M\}$ is $(n+1)^{(M-1) K}$.

A similar argument can be applied for signals intended for Receiver $j$ for all $j \in\{2,3, \ldots, M\}$. Therefore, the received signals can be represented as

$$
\begin{aligned}
& y_{1}=\tilde{y}_{1}+I_{12}+I_{13}+\ldots+I_{1 M}+z_{1}, \\
& y_{2}=\tilde{y}_{2}+I_{21}+I_{23}+\ldots+I_{2 M}+z_{2} \text {, } \\
& \vdots=\quad \vdots \quad \vdots \quad \ddots \quad \vdots \\
& y_{M}=\tilde{y}_{M}+I_{M 1}+I_{M 2}+\ldots+I_{(M-1) M}+z_{1},
\end{aligned}
$$

where $I_{j i}$ is the part of interference caused by all messages intended for Receiver $i$ at Receiver $j$. Due to symmetry, we only consider the received directions at Receiver 1. At Receiver 1, there are $M_{1}$ interfering signals, each of which consists of at most $(n+1)^{(M-1) K}$ directions. Therefore, the total number of interfering directions is $L_{1}^{\prime}=(M-1)(n+1)^{(M-1) K}$. On the other hand, $\tilde{y}_{1}$ consists of $K n^{M-1}(n+1)^{(M-1)(K-1)}$ directions. This is due to the fact that $\tilde{y}_{1}=h_{11}^{2} x_{11}+h_{12}^{2} x_{12}+$ $\ldots+h_{1 K}^{2} x_{1 K}$ and $x_{1 i}$ for all $i \in\{1,2, \ldots, K\}$ consists of $n^{M-1}(n+1)^{(M-1)(K-1)}$ directions. Therefore, the total number of received directions is

$$
L=(M-1)(n+1)^{(M-1) K}+K n^{M-1}(n+1)^{(M-1)(K-1)} .
$$

Using Theorem 6, we can conclude that

$$
r_{\mathrm{Xsum}} \geq \frac{K M n^{M-1}(n+1)^{(M-1)(K-1)}}{K n^{M-1}(n+1)^{(M-1)(K-1)}+(M-1)(n+1)^{(M-1) K}+1}
$$


is achievable for the $X$ channel. By rearranging, we obtain

$$
r_{\text {Xsum }} \geq \frac{K M}{K+(M-1)\left(\frac{n+1}{n}\right)^{M-1}+\frac{1}{n^{M-1}(n+1)^{(M-1)(K-1)}}} .
$$

Since (57) holds for all $n$, we obtain

$$
r_{\text {Xsum }}=\frac{K M}{K+M-1},
$$

which is the desired result. In a special case, $M=K$ and the total DOF is $\frac{K^{2}}{2 K-1}$. This shows that as the number of transmitter and receivers increases the DOFs of $X$ and GIC behaves similarly.

\section{CONCLUSION}

In this paper, we have considered three static channels, namely the $K$-user Gaussian Interference Channel (GIC), the uplink channel of cellular systems, and the $K \times M X$ channel. We have proved that the total DOF of these systems can be attained by incorporating real interference alignment in the signaling. We have proved that single antenna systems can be treated similar to multiple antenna systems where directions can be used for data transmission and reception. This result is obtained by proposing a new coding scheme in which several fractional dimensions are embedded into a single real line. These fractional dimensions play the role of integral dimensions in Euclidean spaces. This fact is supported by a recent extension of Khintchine-Groshev theorem for the non-degenerate manifolds. The total DOF of the MIMO case as well as the complex case is also achieved by a simple application of the main result.

\section{REFERENCES}

[1] C. E. Shannon, "Two-way communication channels," Proc. 4th Berkeley Symp. on Mathematical Statistics and Probability, vol. 1, pp. 611-644, 1961.

[2] M. A. Maddah-Ali, A. S. Motahari, and A. K. Khandani, "Communication over MIMO $X$ channels: Interference alignment, decomposition, and performance analysis," Information Theory, IEEE Transactions on, vol. 54, no. 8, pp. 3457-3470, August 2008.

[3] T. S. Han and K. Kobayashi, "A new achievable rate region for the interference channel," IEEE Trans. Inf. Theory, vol. IT-27, pp. 49 - 60, Januray 1981.

[4] R. Etkin, D. Tse, and H. Wang, "Gaussian interference channel capacity to within one bit," Information Theory, IEEE Transactions on, vol. 54, no. 12, pp. 5534-5562, December 2008.

[5] A. Motahari and A. Khandani, "Capacity bounds for the Gaussian interference channel," Information Theory, IEEE Transactions on, vol. 55, no. 2, pp. 620 - 643, February 2009.

[6] X. Shang, G. Kramer, and B. Chen, "A new outer bound and the noisyinterference sum-rate capacity for Gaussian interference channels," Information Theory, IEEE Transactions on, vol. 55, no. 2, pp. 689-699, February 2009.

[7] V. S. Annapureddy and V. V. Veeravalli, "Sum capacity of the Gaussian interference channel in the low interference regime," roceedings of ITA Workshop, San Diego, CA, January 2008.

[8] O. O. Koyluoglu, H. E. Gamal, L. Lai, and H. V. Poor, "Interference alignment for secrecy," http://arxiv.org/abs/0810.1187, 2008.

[9] S. A. Jafar and S. Shamai, "Degrees of freedom region of the MIMO $X$ channel," Information Theory, IEEE Transactions on, vol. 54, no. 1, pp. 151-170, 2008.

[10] V. R. Cadambe and S. A. Jafar, "Interference alignment and degrees of freedom of the $K$-user interference channel," Information Theory, IEEE Transactions on, vol. 54, no. 8, pp. 3425-3441, 2008.

[11] — " "Degrees of freedom of wireless $X$ networks," IEEE International Symposium on Information Theory, pp. 1268-1272, July 2008.
[12] B. Nazer, M. Gastpar, S. A. Jafar, and S. Vishwanath, "Ergodic interference alignment," http://arxiv.org/abs/0901.4379, 2009.

[13] G. Bresler, A. Parekh, and D. Tse, "The approximate capacity of the many-to-one and one-to-many Gaussian interference channels," http://arxiv.org/abs/0809.3554, 2008.

[14] S. Sridharan, A. Jafarian, S. Vishwanath, and S. A. Jafar, "Capacity of symmetric K-user Gaussian very strong interference channels,' http://arxiv.org/abs/0808.2314, 2008.

[15] S. Sridharan, A. Jafarian, S. Vishwanath, S. A. Jafar, and S. Shamai, "A layered lattice coding scheme for a class of three user Gaussian interference channels," http://arxiv.org/abs/0809.4316, 2008.

[16] R. Etkin and E. Ordentlich, "On the degrees-of-freedom of the K-user Gaussian interference channel," http://arxiv.org/abs/0901.1695, 2009.

[17] A. S. Motahari, S. O. Gharan, and A. K. Khandani, "On the degrees-offreedom of the three-user gaussian interfererence channel: The symmetric case," Presented at IEEE International Symposium on Information Theory, July 2009.

[18] - "Real interference alignment with real numbers," http://arxiv.org/abs/0908.1208, August 2009.

[19] M.A. Maddah-Ali, "On the Degrees of Freedom of the Compound MIMO Broadcast Channels with Finite States," http://arxiv.org/abs/0909.5006, September 2009.

[20] C. Huang and S. A. Jafar, "Degrees of freedom of the mimo interference channel with cooperation and cognition," http://arxiv.org/abs/0803.1733. 2008.

[21] A. Ghasemi, A.S. Motahari, and A.K. Khandani, "Interference Alignment for the $K$ User MIMO Interference Channel," http://arxiv.org/abs/0909.4604. 2009.

[22] W. M. Schmidt, Diophantine approximation. Berlin, Springer-Verlag, 1980.

[23] G. H. Hardy and E. M. Wright, "An introduction to the theory of numbers," fifth edition, Oxford science publications, 2003.

[24] V. Bernik, D. Kleinbock, and G. Margulis, "Khintchine-type theorems on manifolds: the convergence case for standard and multiplicative versions," International Mathematics Research Notices, no. 9, p. 453486, 2001.

[25] V. Beresnevich, "A groshev type theorem for convergence on manifolds," Acta Mathematica Hungarica 94, no. 1-2, pp. 99-130, 2002. 